UW/PT 95-07

\title{
Electroweak Baryogenesis in Supersymmetric Models
}

\author{
Patrick Huet, Ann E. Nelson \\ Department of Physics \\ University of Washington, Box 351560 \\ Seattle, WA 98195-1560
}

The baryon density which may be produced during the electroweak phase transition in supersymmetric models is computed, taking into account the previously neglected effects of transport, strong and weak anomalous fermion number violation, thermal scattering, and a new method for computing $C P$ violating processes during the transition. We can account for the observed baryon asymmetry, provided new $C P$-violating phases are greater than $\sim 10^{-(2-4)}$, and some superpartners are light enough to be relevant during the transition, which takes place at a temperature of (50-100) GeV. In one case, light superpartners are the top squarks and the charginos and/or the neutralinos; in another case the top squarks and both Higgs doublets are light. Our calculation is easily extended to the case of a general two Higgs model, where we find sufficient baryogenesis provided that a certain combination of parameters in the Higgs potential leads to a $C P$ violating space dependent phase in the top quark mass of order $10^{-3}$.

10/95 (revised version) 


\section{Introduction}

Supersymmetry is an attractive candidate for the physics of electroweak symmetry breaking, while electroweak baryogenesis (EWB) [1] is an explanation of the origin of the cosmological asymmetry between matter and antimatter in terms of experimentally accessible physics. It is therefore of interest to understand whether EWB is feasible in supersymmetric models. The only previous estimates [2, 3] of the baryon asymmetry produced in supersymmetric models neglected many effects which are now understood to be important, such as transport [4] and thermal scattering [7]-9].

Let us review the physics relevant for EWB. Anomalous baryon violation in the weak interactions takes place via unobservably slow tunnelling processes at zero temperature [10], but at temperatures above the critical temperature for the weak phase transition, theoretical estimates give a rate $\Gamma=\kappa \alpha_{w}^{4} T$, where $\alpha_{w}$ is the weak fine structure constant [11], and $\kappa$ is a pure number of order ond. Thus electroweak baryon number violation is fast enough in the early universe to change the cosmological baryon number. In thermal equilibrium, unless some nonanomalous approximately conserved quantum number is nonzero [14], anomalous processes will wash out any net baryon number, however a first order electroweak phase transition can provide the departure from thermal equilibrium necessary to generate a nonzero baryon number. Electroweak baryogenesis is only feasible if two conditions are met, which probably require new weak scale physics beyond the Minimal Standard Model (MSM) 22,3,15, [19]. (For relatively recent reviews, see [20].)

1. The transition must be strongly enough first order so that after the transition the anomalous baryon number violation is too slow to wash out the baryons created during the transition [21]. This rate is proportional to $\exp \left(-M_{s} / T\right)$, where $M_{s}$, the energy of the sphaleron field configuration, is proportional to the $W$ boson mass, $M_{s}=$ $(90-160) M_{w}$ [22]. The condition that the $W$ mass jumps to a large enough value during the transition to avoid post-transition baryon number washout requires a light Higgs in the MSM [18,23,24] (in lattice simulations, the transition appears too weakly

1 An early estimate of $\kappa$ gave $\kappa \gtrsim 0.112$ and a recent computation claims a value of $\kappa=$ $1.09 \pm 0.04[13]$. 
first order unless $m_{H} \ll M_{w}$ [25]). However with a top mass of (170-200) GeV, if the MSM is valid up to $10^{6} \mathrm{GeV}$ we will only be in the MSM ground state today for a Higgs mass heavier than $\sim M_{w}[26]$.

2. The amount of $C P$ violation must be just right to explain the observed baryon to entropy ratio, $n_{B} / s \sim 10^{-10}$. The $C P$ violation in the minimal standard model is only physical in processes which involve all the Cabbibo-Kobayashi-Maskawa (CKM) angles and in which all the like charge quark mass differences play a role, which makes it seem a priori difficult for Kobayashi-Maskawa $C P$ violation to generate sufficient baryon number during the weak transition. An interesting attempt to find a large enhancement of the CKM contribution to electroweak baryogenesis was made by Farrar and Shaposhnikov [27] but was later shown not to work due to quantum decoherence effects [7,9].

In constrast to the MSM case, in most extensions of the standard model there can be additional sources of $C P$ violation which appear in particle mass matrices. During a first order electroweak phase transition, bubbles of the broken phase nucleate and expand. Inside the bubble wall, particle mass matrices acquire nontrivial space-time dependence and cannot be made real and diagonal at all points without introducing new $C P$ violating terms into the particle dispersion relations. In a recent paper [8] we introduced a general method for computing the effects of the $C P$ violating mass terms on particle distributions, which takes into account both the effects of scattering from thermal particles and the terms which lead to $C P$ violation in particle propagation. It is now established that transport of $C P$ violating quantum numbers into the symmetric phase, where anomalous electroweak baryon number violation is relatively rapid, plays a dominant role in electroweak baryogenesis for all bubble wall widths [4,6,15, 16].

The most well motivated viable theories for weak scale baryogenesis are two (or more) Higgs models [28] and models with weak scale supersymmetry. In the two Higgs model the relevant $C P$ violation is produced by a phase in the Higgs potential, which leads to $C P$ violating mass matrices for fermions and Higgs bosons, and produces especially large $C P$ violating effects on the Higgs and axial top number distributions. Experimental constraints on atomic and neutron dipole moments allow the relevant phase to be as large as $\mathcal{O}(1)$ 
[29]. Also, the two Higgs model can easily simultaneously satisfy the constraints on Higgs particle masses and the requirement of a sufficiently first order transition [28, 30, 31]. There are many possible supersymmetric extensions of the MSM, with additional $C P$-violating phases. The minimal additional particle content (the Minimal Supersymmetric Standard Model, or MSSM) includes superpartners for all particles and a second Higgs doublet. The supersymmetric terms in the Lagrangian do not introduce any additional $C P$ violation, however supersymmetry must be broken by adding soft supersymmetry breaking operators, which in general are $C P$ violating. If the new $C P$ violating phases are of order one, the neutron and atomic electric dipole moments are larger than the experimental bounds [32] unless the superpartners are unnaturally heavy [33], hence the usual assumption is that the soft supersymmetry breaking terms arise from $C P$ conserving physics and have negligible phases. However it has recently been argued [34] that in most grand unified supersymmetric theories, renormalization of the soft operators between the Planck mass and the scale of grand unification will induce phases of order $10^{-2}-10^{-3}$ in the soft susy breaking operators, providing a new source of $C P$-violation into the low energy effective theory, which is just beyond our current experimental reach. In this paper we will assume that the supersymmetry breaking terms have $C P$-violating phases and see whether these phases can account for sufficient baryogenesis without violating the electric dipole moment bounds[35]. In the MSSM, the requirement of a sufficiently first order phase transition places upper limits on the Higgs and stop (supersymmetric partners of the top) masses [36] which are barely consistent with experimental constraints-there are speculations that these bounds could be relaxed slightly by higher order and nonperturbative effects $[37]^{2}$. The MSSM may easily be extended by adding a gauge singlet [38] which substantially removes these constraints. Here we will consider models both with and without a singlet, but we will only consider those sources of additional $C P$ violation which may be present in the MSSM, with a general set of soft supersymmetry breaking terms consistent with experimental bounds. Therefore we will not worry about the mass upper bounds of ref. [36],

2 Note that in supersymmetric models, the vacuum stability lower bounds [26] on the Higgs mass do not apply. 
and we will assume the Higgs potential is $C P$-conserving 3 . We refer to the supersymmetric model either with or without additional gauge singlets as the Supersymmetric Standard Model, or SSM.

In the next section we discuss the dominant baryogenesis mechanisms in the SSM. In $\S 3$ of this paper we write down the set of coupled differential equations which describe particle interactions and transport during the weak phase transition, and make reasonable approximations which allow us to find an analytic solution for the baryon asymmetry in the SSM. In $\S 4$ we do the same for the two Higgs model. We conclude with a summary of our results and their implications in $\S 5$.

\section{2. $C P$ violation and Particle Sources in the SSM}

Following previous work [2, $8,15,19]$ we compute the baryon asymmetry using the following steps:

I. Compute the $C P$-violating perturbations of the plasma locally induced by the passage of the wall ("particle source terms"). In ref. [8] we described all the sources in terms of quantum mechanical $C P$-violating reflection and transmission from layers of the phase boundary, combined with re-thermalization of the phase-space distributions. Unlike earlier calculations, whose applicability was restricted to either a "thin wall" or a "thick wall", referring to whether the wall thickness is larger or smaller than the relevant mean free paths, our approach provides a unified and consistent treatment for all values of the wall thickness. The proposed method links the charge generation to microphysical CP violating processes, and hence can be widely applied. It generalizes the method developed in Ref. [7] and so properly incorporates decoherence effects which have been shown to have a major negative impact on the generation of a $C P$ violating observable in the MSM [7, 9].

II. We approximate the solution to the Boltzmann equations for particle distribution functions by writing down and solving a set of coupled differential equations for the

3 In some models with a singlet there can be $C P$-violation in the Higgs potential which can produce $C P$-violating effects very similar to those in two Higgs models, however in most models the Higgs potential automatically conserves $C P$. 
local particle densities including the source terms, transport, Debye screening 39] of induced gauge charges 1 and particle number changing reactions [5]. The solution to these equations generally includes a net baryon number, which is produced in the symmetric phase and is transported into the bubbles of broken phase, where it survives until the present provided that the phase transition is sufficiently first order.

In this section we focus on the first step in the calculation. $C P$-violating particle source terms have been shown to result for a selected subset of species in the plasma which mix with one another via a mass matrix with complex phases which either:

a) cannot be rotated away as the result of interactions with the plasma [27.

b) cannot be rotated away at two adjacent points $x$ and $x+d x$, by the same set of unitary transformations, that is, $U_{x}^{-1} U_{x+d x} \neq 1$.

When present, the second mechanism dominates over the first one, as the first mechanism generically involves additional particles whose coupling to the plasma, yields further suppressions. It is the second mechanism which controls baryon generation in the SSM as the neutralinos, charginos, and squarks, have mass matrices with $C P$ violating entries and a non-trivial space-dependence due to the Higgs vacuum expectations values ( $c f . \S 2.2)$. So is the case in the two Higgs models with explicit $C P$ violation in the Higgs potential which yields a space dependent phase to the top quark and Higgs masses in the minimal standard model, the quark mass matrix has only an overall dependence on the Higgs vacuum expectation value and can be diagonalized by space-independent unitary rotations, hence, it can generate a $C P$ violating observable through mechanism $a$ ) rather than through mechanism b) - i.e.through charge current interactions which correct the dispersion relation of the propagating quark in the plasma [27]. This mechanism, however, has been shown to be quite ineffective at generating a significant baryon asymmetry in the MSM [7, 9].

4 In practice we simplify our equations by ignoring the effects of screening since the impact on baryogenesis turns out to be of order 1 40,41.

5 Another potentially relevant species in this model is the $\tau$-lepton, and some have argued that its contribution dominates that of the top quark and Higgs [31, 42]. We do not confirm the importance of the $\tau$ lepton unless $\tan \beta$ is very large. 
The method introduced in Ref. [8] can account for both mechanisms. However, as we are concerned with extensions of the standard model for which the second mechanism $b$ ) is dominant, we will review the general principles of the method for this specific situation 6 .

\subsection{The Method}

Let us consider a set of particles with mass matrix $M(z)$ and moving, in the rest frame of the wall, with energy-momentum $E, \vec{k}$. We wish to find the $C P$-violating asymmetry in their distributions which results from their passage across the wall. We define $z_{o}$ to be their last scattering point, where they emerge from a thermal ensemble with a probability distribution represented by a density matrix $\rho_{z_{o}}$. These particles propagate freely during a mean free time $\tau$, then rescatter and return to the local thermal ensemble in the plane $z_{o}+\tau v, v$ being the velocity perpendicular to the wall, $k_{\perp} / E$. During the time $\tau$, these particles evolve according to a set of Klein-Gordon, Majorana or Dirac equations coupled through the mass matrix $M(z)$. (Some effects of interaction with the plasma, which do not destroy quantum coherence, can easily be included in these equations.) In the course of this evolution $C P$ violation affects the distribution of these particles. At $z_{o}$, the contribution of these particles to any given charge cancel exactly the contribution of their antiparticles since the charge is $C P$ odd and we take the density matrix to be $C P$ even. However, after evolving a time $\tau$ across the $C P$ violating space-dependent background, this cancellation no longer takes place. At the subsequent scattering point $z_{o}+\tau v$, these charges assume a non-zero value, as the evolution of the particles over the distance $\tau v$ can be $C P$-violating. Specifically, the probability for a particle emitted at $z_{0}$ to be transmitted to $z_{0}+\tau v$ can be different from the transmission probability for its $C P$ conjugate. It is only necessary to follow the contribution of a selected subset of charges carried by these particles in order to characterize completely the departure from thermal equilibrium resulting from the passage of the wall. In this subset, there are charges which are explicitly violated by the mass matrix (axial charge ... ) and charges which are exactly conserved ( B-L, ...). In the latter case, there is no net charge density created but, instead, a net spatial current

6 Our method with mechanism $a$ ), applied to the standard model, would give results in agreement with the ones obtained in ref. [7], where similar techniques have been used. 
emerges: opposite charges move in opposite directions. This spatial current arises at high order in masses and the wall velocity $v_{w}$ and, in most of the cases we considered, yield a subleading contribution to the baryon asymmetry. We are mostly interested in the former charges, that is, those charges which are violated by the presence of the wall. In addition to a spatial current, those charges develop a net average density; the latter is linear in the wall velocity and arises at low order in the mass expansion. We will focus our attention on those charges which develop a net density. We alert the reader that there may be situations in which conserved charges have to be equally considered.

To be more quantitative, we introduce $J_{ \pm}$, the average current resulting from particles moving toward positive(negative) $z$ between $z_{o}$ and $z_{o}+\Delta$, where

$$
\Delta \equiv \tau v
$$

The current $J_{+}$receives contributions from either particles originating from the thermal ensemble at point $z_{o}$, moving with a positive velocity and being transmitted at $z_{o}+\Delta$, or from particles originating at $z_{o}+\Delta$, moving with velocity $-v$ and being reflected back towards $z_{o}+\Delta$ (Fig. 1a). A similar definition exists for $J_{-}$(Fig. 1b). $J_{ \pm}$are $C P$ violating currents which are associated with each layer of thickness $\Delta$ moving along with the wall; they can be computed according to

$$
\begin{aligned}
& J_{+}=\left\{\operatorname{Tr} \rho_{z_{o}}\left[T^{\dagger} \hat{Q} T-\bar{T}^{\dagger} \hat{Q} \bar{T}\right]+\operatorname{Tr} \rho_{z_{o}+\Delta}\left[\tilde{R}^{\dagger} \hat{Q} \tilde{R}-\overline{\tilde{R}}^{\dagger} \hat{Q} \overline{\tilde{R}}\right]\right\}\left(\begin{array}{c}
1 \\
0 \\
0 \\
\tilde{v}
\end{array}\right) \\
& J_{-}=\left\{\operatorname{Tr} \rho_{z_{o}}\left[R^{\dagger} \hat{Q} R-\bar{R}^{\dagger} \hat{Q} \bar{R}\right]+\operatorname{Tr} \rho_{z_{o}+\Delta}\left[\tilde{T}^{\dagger} \hat{Q} \tilde{T}-\overline{\tilde{T}}^{\dagger} \hat{Q} \overline{\tilde{T}}\right]\right\}\left(\begin{array}{c}
1 \\
0 \\
0 \\
-v
\end{array}\right)
\end{aligned}
$$

$R(\bar{R})$ and $T(\bar{T})$ are reflection and transmission matrices of particles(anti-particles) produced at $z_{o}$ with a probability matrix $\rho_{z_{o}}$, evolving toward positive $z$ (increasing mass); $\tilde{R}$ and $\tilde{T}$ are the corresponding quantities for particles produced at $z_{o}+\Delta$ with probabilities contained in $\rho_{z_{o}+\Delta}$ and evolving toward negative $z ; v$ is the magnitude of the group velocity perpendicular to the wall at point $z_{o}$ while $\tilde{v}$ is the same quantity but a distance $\Delta$ away. 
Finally, $\hat{Q}$ is the operator corresponding to the chosen charge and the trace is taken over all relevant degrees of freedom and averages over the location $z_{o}$ within a layer of thickness $\Delta$.

Formulae (2.2) provide a concise method of computing the $C P$ violating charge currents $J_{ \pm}$, which results from the propagation and the mixing of particles within the layer of thickness $\Delta$ at point $z_{o}$. After a boost to the plasma frame, these currents constitute the fundamental $C P$ violating building blocks that we need to construct the source terms of the system of rate equations introduced in $\S 3$, which ultimately will convert them through diffusion and relaxation mechanisms into a net baryon asymmetry.

For our purpose, we construct the source terms as follows. Consider a small volume element in the plasma. As the wall crosses it, it deposits into it the current density $\left(J_{+}+J_{-}\right)_{\text {plasma }}^{\mu}$ every time interval $\tau$; the subscript $)_{\text {plasma }}$ refers to the quantity boosted to the plasma frame. At an arbitrary time $t$, the current density accumulated by this mechanism is

$$
s^{\mu}=\int_{t-\tau_{R}}^{t} \frac{1}{\tau} d t^{\prime}\left(J_{+}\left(\vec{x}, t^{\prime}\right)+J_{-}\left(\vec{x}, t^{\prime}\right)\right)_{\text {plasma }}^{\mu} .
$$

Here, $\tau_{R}$ is a typical relaxation time. From this, we infer the net rate of change of charge $Q$ per unit volume to be

$$
\begin{aligned}
\gamma_{Q}(\vec{x}, t)= & \partial_{\mu} s^{\mu} \\
= & \frac{1}{\tau}\left(J_{+}(\vec{x}, t)+J_{-}(\vec{x}, t)\right)_{\text {plasma }}^{0}-\frac{1}{\tau}\left(J_{+}\left(\vec{x}, t-\tau_{R}\right)+J_{-}\left(\vec{x}, t-\tau_{R}\right)\right)_{\text {plasma }}^{0} \\
& -\int_{t-\tau_{R}}^{t} \frac{1}{\tau} \partial_{z}\left(J_{+}+J_{-}\right)_{\text {plasma }}^{z} .
\end{aligned}
$$

Formula (2.3) along with formulae (2.2) constitute the starting point for our analysis of the SSM and of the two Higgs model. In practice, we simplify eq. (2.4) by making an expansion in spatial derivatives, allowing us to neglect the third term, and we take $\tau_{R}$ large, so that we can neglect the second term, whose effect is accounted for as an independent relaxation term in our rate equations (cf. §3).

7 We leave aside diffusion which is accounted for independently in the rate equations. 
One particular advantage of the method above is that it does not require any assumption about the relative magnitude of the mean free paths and the thickness of the wall. Hence, in contrast with earlier methods, it unifies all electroweak baryogenesis scenarios.

\subsection{The $S S M$}

In the SSM, we are interested in the generation of charges which (a) are approximately conserved in the unbroken phase so that, they can diffuse a long way in front of the bubble wall, where anomalous baryon violation is fast and (b) are non-orthogonal to baryon number, so that their relaxation energetically favors a non-zero baryon charge. Candidates of choice are Higgs number and axial top number. The generation of these charges results from the mixing of the charginos, neutralinos and the mixing of top squarks respectively. The chargino mass matrix is, in the basis $\tilde{W}_{+}, \tilde{W}_{-}, \tilde{h}_{-}, \tilde{h}_{+}^{\prime}$

$$
\mathcal{M}_{\tilde{c}}=\left(\begin{array}{cccc}
0 & \tilde{m}_{2} & -v_{2} & 0 \\
\tilde{m}_{2} & 0 & 0 & -v_{1} \\
-v_{2} & 0 & 0 & e^{i \phi_{B}} \mu \\
0 & -v_{1} & e^{i \phi_{B}} \mu & 0
\end{array}\right)
$$

with $v_{1}=\sqrt{2} M_{W}(z, T) \cos \beta(z)$ and $v_{2}=\sqrt{2} M_{W}(z, T) \sin \beta(z) ; \quad M_{W}(z, T)$ is the temperature-dependent $W$ mass defined at each point $z$ in the wall. The neutralinos mass matrix is, in the basis $\tilde{W}_{3}, \tilde{B}, \tilde{h}_{0}, \tilde{h}_{0}^{\prime}$,

$$
\mathcal{M}_{\tilde{n}}=\left(\begin{array}{cccc}
\tilde{m}_{2} & 0 & u_{2} \cos \theta_{w} & -u_{1} \cos \theta_{w} \\
0 & \tilde{m}_{1} & -u_{2} \sin \theta_{w} & u_{1} \sin \theta_{w} \\
u_{2} \cos \theta_{w} & -u_{2} \sin \theta_{w} & 0 & -e^{i \phi_{B}} \mu \\
-u_{1} \cos \theta_{w} & u_{1} \sin \theta_{w} & -e^{i \phi_{B}} \mu & 0
\end{array}\right)
$$

with $u_{1}=M_{Z}(z, T) \cos \beta(z)$ and $u_{2}=M_{Z}(z, T) \sin \beta(z)$. Finally, in the basis $\tilde{t}_{L}, \tilde{t}_{R}$,

$$
\mathcal{M}_{\tilde{t}}=\left(\begin{array}{cc}
\tilde{m}_{L}^{2} & a^{2} e^{i \alpha} \\
a^{2} e^{-i \alpha} & \tilde{m}_{R}^{2}
\end{array}\right)
$$

here, we defined $a^{2} e^{i \alpha}=\sqrt{2} \frac{\lambda_{t}}{g}\left(A e^{i \phi_{A}} v_{2}+\mu e^{i \phi_{B}} v_{1}\right)$.

With these conventions, the charge operator for the Higgs number takes the form

$$
\hat{Q}_{\tilde{h}}=\operatorname{Diag}(0,0,1,-1),
$$


while for the axial stop number, the charge operator is defined to be

$$
\hat{Q}_{\tilde{s}}=\operatorname{Diag}\left(\frac{1}{2},-\frac{1}{2}\right) .
$$

We can now compute sources for those charges. We choose to perform an expansion in powers of mass. This expansion, introduced in [7] and further developed in [8], is adequate to demonstrate the quantum mechanical physics required for generating a $C P$ violating observable. In particular, it generates polynomials in $\mathcal{M}$ whose imaginary part of the trace yields an expansion in terms of $C P$ violating invariants 8 . We will discuss the validity of this approximation later on in this section.

- Charginos and Neutralinos -

In order to compute the source $\gamma_{\tilde{h}}(\vec{x}, t)$ for the Higgsino number, we begin by computing the corresponding current sources $J_{ \pm}$as given in Eqs. (2.2). Their determination requires the knowledge of transmission and reflection amplitudes which are obtained by solving a set of coupled Majorana equations with the chargino and neutralino mass matrices given in (2.5)-(2.6). We obtain up to an overall phase, at leading order in $\mathcal{M}_{(\tilde{c}, \tilde{n})}^{2}$,

$$
\begin{aligned}
T=1-\int_{0}^{\Delta} d z_{1} \int_{0}^{z_{1}} d z_{2} \mathcal{M}_{2} \mathcal{M}_{1}^{*} e^{i 2 \omega\left(z_{1}-z_{2}\right)} & \\
+ & \int_{0}^{\Delta} d z_{1} \int_{0}^{z_{1}} d z_{2} \int_{z_{2}}^{\Delta} d z_{3} \int_{0}^{z_{3}} d z_{4} \mathcal{M}_{4} \mathcal{M}_{3}^{*} \mathcal{M}_{2} \mathcal{M}_{1}^{*} e^{i 2 \omega\left(z_{1}-z_{2}+z_{3}-z_{4}\right)}+\ldots \\
\tilde{R} & =\int_{0}^{\Delta} d z_{1} \mathcal{M}_{1} e^{-i 2 \omega z_{1}} \\
& +\int_{0}^{\Delta} d z_{2} \int_{z_{2}}^{\Delta} d z_{3} \int_{0}^{z_{3}} d z_{4} \mathcal{M}_{4} \mathcal{M}_{3}^{*} \mathcal{M}_{2} e^{i 2 \omega\left(-z_{2}+z_{3}-z_{4}\right)}+\ldots
\end{aligned}
$$

and similar expressions for $\tilde{T}, R$. The quantity $\omega$ stands for the magnitude of the energy of motion transverse to the wall, and $\mathcal{M}_{i}$ is short for $\mathcal{M}_{(\tilde{c}, \tilde{n})}\left(z_{i}\right)$. The leading $C P$ violating contributions arise at order $\mathcal{O}(\mathcal{M} / T)^{4}$. Contributing paths are depicted in Fig. 2.

In order to use Eq. (2.2) to compute $J_{ \pm}$, we need the density matrices $\rho_{z_{o}}$ and $\rho_{z_{o}+\Delta}$, describing the distribution of particles in phase and flavor spaces at their production point

8 There are also $C P$ violating self-energy corrections, which are the main source of $C P$ violation in the absence of the one considered here. 
$z_{o}$ and $z_{o}+\Delta$. We choose the density matrices describing equilibrium distributions of mass eigenstates in the unbroken phase

$$
\begin{aligned}
& \rho_{z_{o}}^{n}=\operatorname{Diag}\left(n_{\tilde{m}_{2}}(E, \tilde{v}), n_{\tilde{m}_{1}}(E, \tilde{v}), n_{\mu}(E, \tilde{v}), n_{\mu}(E, \tilde{v})\right) \\
& \rho_{z_{o}}^{c}=\operatorname{Diag}\left(n_{\tilde{m}_{2}}(E, \tilde{v}), n_{\tilde{m}_{2}}(E, \tilde{v}), n_{\mu}(E, \tilde{v}), n_{\mu}(E, \tilde{v})\right)
\end{aligned} .
$$

We construct $\rho_{z_{o}+\Delta}^{c, n}$ from $\rho_{z_{o}}^{c, n}$ with the substitution $\tilde{v} \leftrightarrow-v$. In these expressions, $n_{m}(E, \tilde{v})$ is the Fermi-Dirac distribution, $n_{f}$, of a species of mass $m$, boosted to the wall frame,

$$
n_{m}=\left(\operatorname{Exp}\left[\gamma_{w}\left(E-v_{w} k_{\perp}\right)\right]+1\right)^{-1}
$$

Some motivations for our choice of density matrix are as follows. In a regime of large masses $\tilde{m}_{2}, \tilde{\mu} \geq T$, it is obviously sensible to assume that $\rho$ is diagonal in the mass eigenstate basis; in a regime of small masses, $\leq T$, particles are produced as interaction eigenstates which differ from mass eigenstates by a unitary rotation; ignoring this rotation amounts to ignoring small corrections of order $(\mathcal{M} / T)^{2}$. Furthermore, the choice of a thermal distribution is reasonable as the non-equilibrium component of the distributions is of order $v_{w}$, that is, it amounts to ignoring terms of order $v_{w}^{2} \leq 1$ [24,43]. Inserting Eqs. (2.10) and (2.11) in Eq. (2.2) yields

$$
\begin{aligned}
J_{+}=(1,0,0, \tilde{v}) \times & \left\{\begin{array}{l}
\Delta \\
+
\end{array} \int_{0}^{\Delta} z_{1} \int_{0}^{z_{1}} d z_{2} \int_{z_{2}}^{\Delta} d z_{3} \int_{0}^{z_{3}} d z_{4} \sin 2 \omega\left(z_{4}-z_{3}+z_{2}-z_{1}\right)\right. \\
& \times \operatorname{Im} \operatorname{Tr}\left[\rho_{z_{o}} \mathcal{M}_{1}^{*} \mathcal{M}_{2} \mathcal{M}_{3}^{*} \mathcal{M}_{4} \hat{Q}_{\tilde{h}}\right] \\
- & 4 \int_{0}^{\Delta} d z_{1} \int_{0}^{\Delta} d z_{2} \int_{z_{2}}^{\Delta} d z_{3} \int_{0}^{z_{3}} d z_{4} \sin 2 \omega\left(z_{4}-z_{3}+z_{2}-z_{1}\right) \\
& \left.\times \operatorname{Im} \operatorname{Tr}\left[\mathcal{M}_{1}^{*} \rho_{z_{o}+\Delta} \mathcal{M}_{2} \mathcal{M}_{3}^{*} \mathcal{M}_{4} \hat{Q}_{\tilde{h}}\right]\right\}
\end{aligned}
$$


and

$$
\begin{aligned}
J_{-}=(1,0,0,-v) \times & \{ \\
- & 4 \int_{0}^{\Delta} d z_{1} \int_{z_{1}}^{\Delta} d z_{2} \int_{0}^{z_{2}} d z_{3} \int_{z_{3}}^{\Delta} d z_{4} \sin 2 \omega\left(z_{4}-z_{3}+z_{2}-z_{1}\right) \\
& \times \operatorname{Im} \operatorname{Tr}\left[\rho_{z_{o}+\Delta} \mathcal{M}_{1}^{*} \mathcal{M}_{2} \mathcal{M}_{3}^{*} \mathcal{M}_{4} \hat{Q}_{\tilde{h}}\right] \\
+ & 4 \int_{0}^{\Delta} d z_{1} \int_{0}^{\Delta} d z_{2} \int_{0}^{z_{2}} d z_{3} \int_{z_{3}}^{\Delta} d z_{4} \sin 2 \omega\left(z_{4}-z_{3}+z_{2}-z_{1}\right) \\
& \left.\times \operatorname{Im} \operatorname{Tr}\left[\mathcal{M}_{1}^{*} \rho_{z_{o}} \mathcal{M}_{2} \mathcal{M}_{3}^{*} \mathcal{M}_{4} \hat{Q}_{\tilde{h}}\right]\right\} .
\end{aligned}
$$

In order to proceed with analytic expressions, we simplify further by performing a derivative expansion: $\mathcal{M}(z)=\mathcal{M}\left(z_{o}\right)+\left(z-z_{o}\right) \partial_{z} \mathcal{M}\left(z_{o}\right)+\mathcal{O}(\tau / w)^{2}$ and $v=\tilde{v}+\mathcal{O}(\tau / w)^{2}$. This expansion is only justified in a region of the parameter space for which the mean free time $\tau$ is smaller than the scale of variation of the masses, i.e., the wall thickness $w$. We will discuss the validity of our approximations in the last section. This simplification allows us to perform the trace in flavor space and in phase space independently and yields after summing the contributions from charginos and neutralinos, in first order in the wall velocity $v_{w}$,

$$
\begin{aligned}
& \left(J_{+}+J_{-}\right)^{z}=0 \\
& \left(J_{+}+J_{-}\right)^{0}=\gamma_{w} v_{w} \times \sum_{i}^{\text {eigen. }} \mathcal{J}^{i} \times \int \frac{d^{3} \vec{k}}{(2 \pi)^{3}} \frac{f\left(\omega_{i} \Delta\right)}{\omega_{i}^{5}}\left(2 v_{i}\right) \frac{e^{E_{i} / T}}{\left(1+e^{E_{i} / T}\right)^{2}} \frac{E_{i}}{T}
\end{aligned}
$$

with $f(\xi)$ defined as

$$
f(\xi)=(\xi \cos \xi-\sin \xi)^{2}
$$

and the summation is over the mass eigenstates with eigenvalues $m_{i}$ in the unbroken phase, with $\mathcal{J}^{i}$, a corresponding $C P$-violating invariant. For the charginos, there are two mass eigenstates with masses $\mu$ and $\tilde{m}_{2}$, whose $C P$-violating invariants are

$$
\begin{aligned}
\mathcal{J}_{c h}^{\mu} & =\mathcal{J}_{c h}^{\tilde{m}_{2}}=-\sin \phi_{B} \mu \tilde{m}_{2}\left(v_{2} \partial_{z} v_{1}-v_{1} \partial_{z} v_{2}\right) / T^{5} \\
& =2 \mu \tilde{m}_{2} M_{W}^{2}(T, z) \sin \phi_{B} \partial_{z} \beta / T^{5} .
\end{aligned}
$$


While, for the neutralinos, there are four eigenstates with masses $\mu, \mu, \tilde{m}_{1}$ and $\tilde{m}_{2}$ and with, correspondingly,

$$
\begin{aligned}
\mathcal{J}_{n}^{\mu} & =\left(\mathcal{J}_{n}^{\tilde{m}_{1}}+\mathcal{J}_{n}^{\tilde{m}_{2}}\right) / 2 \\
\mathcal{J}_{n}^{\tilde{m}_{1}} & =-\sin \phi_{B} \mu \tilde{m}_{1} \sin ^{2} \theta_{w}\left(u_{2} \partial_{z} u_{1}-u_{1} \partial_{z} u_{2}\right) / T^{5} \\
& =\mu \tilde{m}_{1} M_{Z}^{2}(T, z) \sin ^{2} \theta_{w} \sin \phi_{B} \partial_{z} \beta / T^{5} \\
\mathcal{J}_{n}^{\tilde{m}_{2}} & =-\sin \phi_{B} \mu \tilde{m}_{2} \cos ^{2} \theta_{w}\left(u_{2} \partial_{z} u_{1}-u_{1} \partial_{z} u_{2}\right) / T^{5} \\
& =\mu \tilde{m}_{2} M_{Z}^{2}(T, z) \cos ^{2} \theta_{w} \sin \phi_{B} \partial_{z} \beta / T^{5} .
\end{aligned}
$$

We can now construct the local source density $\gamma_{\tilde{h}}(\vec{x}, t)$ for the Higgs number by inserting Eq. (2.16) in formula (2.4), we obtain

$$
\begin{gathered}
\gamma_{\tilde{h}}(\vec{x}, t)=\gamma_{w} v_{w} \frac{T^{4}}{4 \pi^{2}}\left(\mathcal{J}_{c h}^{\mu} \mathcal{I}_{\tilde{h}}^{\mu}+\mathcal{J}_{c h}^{\tilde{m}_{2}} \mathcal{I}_{\tilde{h}}^{\tilde{m}_{2}}+2 \mathcal{J}_{n}^{\mu} \mathcal{I}_{\tilde{h}}^{\mu}+\mathcal{J}_{n}^{\tilde{m}_{1}} \mathcal{I}_{\tilde{h}}^{\tilde{m}_{1}}+\mathcal{J}_{n}^{\tilde{m}_{2}} \mathcal{I}_{\tilde{h}}^{\tilde{m}_{2}}\right) \\
+\mathcal{O}\left(v_{w}^{2},(\tau / w)^{2}\right)
\end{gathered}
$$

The factor $\mathcal{I}_{\tilde{h}}^{m}$ contains information on the phase space as well as on effects due to plasma interactions. Its analytic form is

$$
\mathcal{I}_{\tilde{h}}^{m}=\sqrt{\tau T} \int_{\frac{m}{T}}^{\infty} d y y^{2} \frac{e^{y}}{\left(1+e^{y}\right)^{2}} \int_{0}^{\tau T\left(y^{2}-\left(\frac{m}{T}\right)^{2}\right) / y} d \xi \frac{f\left(\sqrt{\xi^{2}+\xi \frac{m_{i}^{2} \tau}{y T}}\right)}{\left(\xi y+\frac{m^{2} \tau}{T}\right)^{5 / 2}}
$$

it is a simple exercise to show that the factor $\mathcal{I}_{\tilde{h}}^{m}$ vanishes rapidly with $\tau$, as

$$
\mathcal{I}_{\tilde{h}}^{m} \propto \tau^{5} \quad(\tau \rightarrow 0)
$$

This steep dependence on $\tau$, simply reflects the high suppression resulting from decoherence due to incoherent scatterings in the plasma whose frequency increases as $1 / \tau$. This suppression has the same origin as the one forbidding electroweak baryogenesis with $C P$ violation originating from the mixing of light quarks in the MSM. For larger coherence time, $\mathcal{I}_{\tilde{h}}^{m}$ scales approximately as

$$
\mathcal{I}_{\tilde{h}}^{m} \propto \frac{1}{\tau T} \quad(\tau \rightarrow \infty)
$$

This fall-off with increasing $\tau$ describes the semi-classical limit in which particles propagate a distance long compared to their Compton wavelength in which case, fast 
oscillations of their wave-function wash away the interference required to generate a $C P$ asymmetry. This fall-off takes place in the thick wall regime, $\tau \leq w$, the situation for which our derivative expansion applies; it persists until $\tau \simeq w$, which defines the thin wall regime. The two behaviors (2.22) and (2.23) are easily identified in Fig. 3.

For the case of interest, the damping rate is essentially dominated by weak interaction processes: $\gamma_{\tilde{h}} \sim \alpha_{w} T$. A crude estimate for the coherence time $\tau_{\tilde{h}}, \sim \gamma_{\tilde{h}}^{-1}$, yields the range $20 / T \leq \tau_{\tilde{h}} \leq 30 / T$, which lies comfortably in the asymptotic domain described by $(2.23)$.

In summary, an analytic form which fits well the source term for the Higgsino number in the domain of interest is Eq. (2.20) with

$$
\mathcal{I}_{h}^{m} \approx \frac{22}{5} \frac{1}{T \tau_{\tilde{h}}} \frac{e^{m / T}}{\left(1+e^{m / T}\right)^{2}}\left(\frac{T}{m}\right)^{5 / 2} \quad 20 / T \leq \tau_{\tilde{h}} \leq 30 / T
$$

This form is only a fit which is valid in the range $0.5<m / T \leq 1$.

Let us assess the domain of validity of the expression above.

1. Results (2.21)-(2.23) are not to be trusted in the limits $\tau>w$ where our derivative expansion does not apply. Instead, in this limit, one expects to observe the dependence on $\tau$ to weaken as $\tau \rightarrow w$, and to vanish as $\tau \gg w$; that is, one expects to observe the factor $\mathcal{I}_{h}^{m}$ to saturate at a value $\tau \sim w$, to become a function of the mass only.

2. The coherence time $\tau_{\tilde{s}}$ given above is an estimate based on our knowledge on the damping rate which, so far, has only been studied in the low momentum limit [44]; this value can only be a crude estimate.

3. Finally, we turn to the important question of the validity of the mass expansion we used to derive formulas (2.20)-(2.23). A careful study of this expansion shows that its expansion parameter is either $m \Delta$ in the limit $\omega \Delta \leq 1$ or $m / \omega$ in the limit $\omega \Delta \geq 1$. In both limits, the expansion parameter is less than one because of the relation $m<\omega=\sqrt{k_{\perp}^{2}+m^{2}}$. Let us give the physical interpretation of those statements9. In a typical scattering off a diffracting medium characterized by a step potential of

9 For a more detailed discussion see Ref. [7]. 
height $V$, reflection and transmission amplitudes result from the constructive and destructive interference of various diffracted waves generated everywhere inside the bulk of the medium. Transmission and reflection amplitudes will be comparable 10 if the incoming wave penetrates coherently the diffracting medium over at least a distance of order $1 / V$, and has few oscillations over that distance. Suppression of the reflection amplitude arises if the coherence length $\Delta$ of the incoming wave is smaller than $1 / V$ or if its energy $\omega$ is larger than $V$. In the case where $\Delta \ll 1 / V$, only a layer $\Delta$ of the medium effectively contributes to the coherent reconstruction of the reflection and transmission amplitudes, this is the phenomenon of decoherence, which suppresses the reflection amplitude with powers of $\Delta V$. In the case where $V \ll \omega$, fast oscillations of the propagating wave inside the medium, tend to attenuate the reconstruction of the reflected amplitude with powers of $V / \omega$. In the present case, the "diffracting medium" is the wall and its height $V$ is the mass $m$ of the scattering particle, hence, suppression factors are controlled by $\Delta m$ or $m / \omega$, whichever is smaller. An alternative method of computation of the currents $J_{ \pm}$consists of computing $R$ and $T$ by solving Majorana equations including the imaginary part of the thermal self-energy. This method automatically accounts for both effects occuring here [7]. In particular, use of the mass expansion in this context suggests an expansion parameter $\sim m \Delta / \sqrt{1+\omega^{2} \Delta^{2}}$, which corroborates the analysis above.

- Squarks -

We now turn to the calculation of the source for the axial stop number. The stop mass matrix is given in (2.7) and the top axial charge operator $\hat{Q}_{\tilde{s}}$ is given in (2.9). As for the Higgsino number, we proceed in computing the current source $J_{ \pm}$in the wall frame using Eqs. (2.2), which we then input into formula (2.4) to construct the source $\gamma_{\tilde{s}}(\vec{x}, t)$.

This time, the amplitudes are computed in solving a set of coupled Klein-Gordon equations. We obtain up to an overall phase, at leading order in $\mathcal{M}_{\tilde{t}}^{2}$,

10 In order to obtain significant $C P$ violating asymmetries, both reflection and transmission amplitudes are to be significantly different from zero, otherwise, as either $|R|$ or $|T|$ goes to zero, the other one goes to one from unitarity, and both $|R|^{2}-|\bar{R}|^{2}$ and $|T|^{2}-|\bar{T}|^{2}$ vanish correspondingly. 


$$
T=\left[1+\ldots-\int_{0}^{\Delta} d z_{1} \int_{0}^{z_{1}} d z_{2} \frac{\mathcal{M}_{2}^{2}}{2 \omega} \frac{\mathcal{M}_{1}^{2}}{2 \omega} e^{i 2 \omega\left(z_{1}-z_{2}\right)}\right]+\ldots
$$

and

$$
\tilde{R}=\ldots+\int_{0}^{\Delta} d z_{1} \frac{\mathcal{M}_{1}^{2}}{2 \omega} e^{-i 2 \omega z_{1}}+\ldots
$$

We only displayed the contributions whose interference contribute to a $C P$ asymmetry in $J_{+}$. These specific paths are depicted in Fig. 4. As we did earlier, we assume the squark density matrices $\rho_{z_{o}}$ and $\rho_{z_{o}+\Delta}$ to describe thermal distributions in the unbroken phase

$$
\begin{aligned}
\rho_{z_{o}}^{s} & =\operatorname{Diag}\left(n_{\tilde{m}_{L}}^{b}(E,+\tilde{v}), n_{\tilde{m}_{R}}^{b}(E,+\tilde{v})\right) \\
\rho_{z_{o}+\Delta}^{s} & =\operatorname{Diag}\left(n_{\tilde{m}_{L}}^{b}(E,-v), n_{\tilde{m}_{R}}^{b}(E,-v)\right)
\end{aligned} .
$$

$n_{m}^{b}(E, \tilde{v})$ now, refers to the Bose-Einstein distribution, $n_{b}$, boosted to the wall frame,

$$
n_{m}^{b}=\left(\operatorname{Exp}\left[\gamma_{w}\left(E-v_{w} k_{\perp}\right)\right]-1\right)^{-1}
$$

The soft supersymmetry breaking masses are kept in (2.27) as, for large values, they yield an exponential suppression of the baryon asymmetry produced.

From (2.25), we obtain for the current sources

$$
\begin{aligned}
J_{+}=(1,0,0, \tilde{v}) & \times\{ \\
& +\int_{0}^{\Delta} d z_{1} \int_{0}^{z_{1}} d z_{2} \frac{\sin 2 \omega\left(z_{1}-z_{2}\right)}{\omega^{2}} \times \operatorname{Im} \operatorname{Tr}\left[\rho_{z_{o}} \hat{Q}_{\tilde{s}} \mathcal{M}_{2}^{2} \mathcal{M}_{1}^{2}\right] \\
& \left.+\int_{0}^{\Delta} d z_{1} \int_{0}^{\Delta} d z_{2} \frac{\sin 2 \omega\left(z_{1}-z_{2}\right)}{2 \omega^{2}} \times \operatorname{Im} \operatorname{Tr}\left[\rho_{z_{o}+\Delta} \mathcal{M}_{2}^{2} \hat{Q}_{\tilde{s}} \mathcal{M}_{1}^{2}\right]\right\}
\end{aligned}
$$

and

$$
J_{-}=J_{+}\left(\rho_{z_{o}} \leftrightarrow \rho_{z_{o}+\Delta}, \tilde{v} \leftrightarrow-v\right)
$$

Performing an expansion in the wall velocity $v_{w}$, we find, in first order in $v_{w}$,

$$
\begin{aligned}
& \left(J_{+}+J_{-}\right)^{z}=0 \\
& \left(J_{+}+J_{-}\right)^{0}=\gamma_{w} v_{w} \times \mathcal{J}_{\tilde{s}} \times \sum_{i=\tilde{m}_{L}, \tilde{m}_{R}} \int \frac{d^{3} \vec{k}}{(2 \pi)^{3}} \frac{g(\omega \Delta)}{4 \omega^{5}}\left(2 v_{i}\right) \frac{e^{E_{i} / T}}{\left(1-e^{E_{i} / T}\right)^{2}} \frac{E_{i}}{T}
\end{aligned}
$$


where $g(\xi)$ is defined as

$$
g(\xi)=1-\cos 2 \xi-\xi \sin 2 \xi
$$

and $\mathcal{J}_{\tilde{s}}$ is a new $C P$ violating invariant given by

$$
\begin{aligned}
\mathcal{J}_{\tilde{s}} & =-\partial_{z} \alpha a^{4} / T^{5} \\
& =4 \frac{\lambda_{t}^{2}}{g^{2}} A \mu \sin \left(\phi_{B}-\phi_{A}\right) M_{W}^{2}(T, z) \partial_{z} \beta .
\end{aligned}
$$

After a few simple manipulations, we derive the following expression for the stop axial source $\gamma_{\tilde{s}}(\vec{x}, t)$,

$$
\begin{aligned}
\gamma_{\tilde{s}}(\vec{x}, t)=\gamma_{w} v_{w} & N_{c} \frac{T^{4}}{4 \pi^{2}} \times \mathcal{J}_{\tilde{s}} \times\left(\mathcal{I}_{\tilde{s}}^{\tilde{m}_{L}}+\mathcal{I}_{\tilde{s}}^{\tilde{m}_{R}}\right) \\
& +\mathcal{O}\left(v_{w}^{2},(\tau / w)^{2}\right)
\end{aligned}
$$

where $N_{c}$ is the number of colors, $=3$. The function $\mathcal{I}_{\tilde{s}}^{m}$ is given by

$$
\mathcal{I}_{\tilde{s}}^{m}=\frac{1}{4} \sqrt{\tau T} \int_{\frac{m}{T}}^{\infty} d y y^{2} \frac{e^{y}}{\left(1-e^{y}\right)^{2}} \int_{0}^{\tau T\left(y^{2}-\left(\frac{m}{T}\right)^{2}\right) / y} d \xi \frac{g\left(\sqrt{\xi^{2}+\xi \frac{m_{i}^{2} \tau}{y T}}\right)}{\left(\xi y+\frac{m^{2} \tau}{T}\right)^{5 / 2}}
$$

it is simple to show that the factor $\mathcal{I}_{\tilde{s}}^{m}$ vanishes rapidly with $\tau$

$$
\mathcal{I}_{\tilde{s}}^{m} \propto \tau^{3} \quad(\tau \rightarrow 0),
$$

as, in this limit, incoherent plasma scatterings become overwhelming. This behavior, already noted in the Higgsino case, is a universal property which can be traced to the quantum nature of $C P$ violation, conflicting with the classical nature of the plasma physics. For larger coherence time, $\mathcal{I}_{\tilde{s}}^{m}$ behaves approximately as 11

$$
\mathcal{I}_{\tilde{s}}^{m} \propto \frac{1}{\tau T} \quad(\tau \rightarrow \infty)
$$

Both behaviors (2.36) and (2.37) are evident on Fig. 5. Unlike the case of the Higgsinos, the squark plasma physics is dominated by strong interactions, and so is the damping rate, hence we estimate $\tau_{\tilde{s}}, \propto\left(2 \alpha_{s} T\right)^{-1}$, to be about $5 / T$. From this estimate, we infer that

11 This behavior is cut off at a value $\tau \sim L$; at this value, $\mathcal{I}_{\tilde{s}}^{m}$ is expected to saturate to its "thin wall" value. 
the regime of relevance is neither the decoherence regime nor the semi-classical regime but rather an intermediate regime corresponding to the peak shown on Fig. 5. This is a situation already encountered in Ref. [8], in the case of the top quark in the two Higgs model (cf. §4).

In summary, and with the above value for $\tau_{\tilde{s}}$, an analytic expression which provides a reliable fit to the source $\gamma_{\tilde{s}}(\vec{x}, t)$ for the axial stop number, in the range $0.5 \leq m / T$, is 12

$$
\gamma_{\tilde{s}}(\vec{x}, t) \simeq \gamma_{w} v_{w} \frac{N_{c} T^{4}}{200 \pi^{2}} \times \mathcal{J}_{\tilde{s}} \times \sum_{m=\tilde{m}_{L}, \tilde{m}_{R}} \frac{T}{m} \frac{e^{m / T}}{\left(1-e^{m / T}\right)^{2}} \quad \text { with } \quad \tau_{\tilde{s}} \simeq 5 / T .
$$

This expression has been derived under the same assumptions as the ones made to derive the corresponding analytic form (2.24) for the source for the Higgsino number, $\gamma_{\tilde{h}}(\vec{x}, t)$. These assumptions have been evaluated in the discussion following Eq. (2.24).

\section{Diffusion equations in the SSM}

Only those particle species which participate in particle number changing transitions which are fast compared with the relevant timescales, but which carry some charge which is approximately conserved in the symmetric phase, can have significant nonzero densities in the symmetric phase during the transition. If the system is near thermal equilibrium and the particles interact weakly, the particle densities $n_{i}$ satisfy

$$
n_{i}=k_{i} \mu_{i} T^{2} / 6
$$

where $\mu_{i}$ is a local chemical potential for particle species $i$, and $k_{i}$ is a statistical factor defined by eq. (3.1). For light, weakly interacting particles $k_{i} \approx 2$ (boson degrees of freedom $)+1$ (fermion degrees of freedom), while for particles much heavier than $T$ it is exponentially small. If we consider a reaction which changes the particle number of particle species $i$ by $\Delta_{i}$, near thermal equilibrium the difference beween the rates for the reaction and its inverse will satisfy

$$
\Gamma_{\text {net }}=\frac{\sum_{i} \Delta_{i} \mu_{i}}{T} \Gamma_{\text {fluct }}=\frac{\sum_{i} n_{i} \Delta_{i}}{k_{i}} 6 \Gamma_{\text {fluct }} / T^{3}
$$

12 We emphasize that this is only a fit. 
where $\Gamma_{\text {fluct }}$ is the total rate for the reaction and its inverse per unit volume. For convenience we will henceforth define particle number changing rates to be $\left(6 / T^{3}\right) \Gamma_{\text {fluct }}$.

We can now write down a set of coupled differential equations which include the effects of diffusion, particle number changing reactions, and $C P$ violating source terms, and solve them to find the various particle densities in the SSM. Anticipating a small departure from equilibrium, we incorporate particle number changing reactions and sources as two distinct terms. Diffusion is described by a standard diffusion term without a provision to account for the potentially fast relative motion of the sources in respect to the plasma. It is a good description in the regime of a wall velocity $v_{w}$ small compared to the speed of sound in the plasma $c_{s}=1 / \sqrt{3}$. This condition, which is likely to be fulfilled in the minimal standard model [24,43], may or may not be fulfilled in more general theories such as the ones considered here. To find out would require a complete calculation of the phase transition, which is beyond the scope of the present work. Further simplifications of these equations take place when we neglect all couplings except for gauge interactions, and the top quark Yukawa coupling. We include the effects of strong sphalerons 45, 46, but neglect the weak sphalerons until near the end of the calculation. The neglect of the weak sphalerons allows us to forget about leptons in our differential equations, and will turn out to be a good approximation when computing Higgs and quark densities. We also neglect the effects of hypercharge gauge forces and screening, which can be shown to affect the baryon number produced by a factor of at most order one [41]. The particle densities we need include $q \equiv\left(t_{L}+b_{L}\right)$, the right handed top quark $t \equiv t_{R}$, the Higgs particles $h \equiv\left(h^{-}+h^{0}+\bar{h}^{\prime+}+\bar{h}^{\prime 0}\right)$, and their superpartners $\tilde{q}, \tilde{t}, \tilde{h}$. The individual particle numbers of these species can change through the top quark Yukawa interaction, the top quark mass, the Higgs self interactions, and anomalous weak interactions, and the supergauge interactions. We will find that baryogenesis in the minimal model is only feasible if some of the superpartners of the gauge and Higgs bosons are light, so that we may take the supergauge interactions to be in thermal equilibrium $\left(q / k_{q}=\tilde{q} / k_{\tilde{q}}, t / k_{t}=\tilde{t} / k_{\tilde{t}}, h / k_{h}=\right.$ $\left.\tilde{h} / k_{\tilde{h}}\right)$, and describe the system by densities $Q=q+\tilde{q}, T=t+\tilde{t}$ and $H=h+\tilde{h}$. As shown in $\S 2, C P$ violating interactions with the phase boundary produce source terms $\gamma_{\tilde{h}}$ for the Higgsinos and $\gamma_{\tilde{s}}$ for the $\tilde{q}-\tilde{t}$ densities, which tend to pull the system away 
from equilibrium. When we include strong sphalerons (with a rate $\Gamma_{s s}$ ), we will generate a right handed bottom quark density, $B \equiv b_{R}+\tilde{b}_{R}$, as well as first and second family quarks $Q_{(1,2) L},, U_{R}, C_{R}, S_{R}, D_{R}$. However since strong sphalerons are the only processes which generate significant numbers of first and second family quarks, and all quarks have nearly the same diffusion constant, we can constrain these densities algebraically in terms of $B$ to satisfy

$$
Q_{1 L}=Q_{2 L}=-2 U_{R}=-2 D_{R}=-2 S_{R}=-2 C_{R}=-2 B=2(Q+T) .
$$

For simplicity we will also assume all squark partners of the light quarks are degenerate and take

$$
k_{Q_{1 L}}=k_{Q_{2 L}}=2 k_{S_{R}}=2 k_{D_{R}}=2 k_{U_{R}}=2 k_{C_{R}}=2 k_{B}
$$

We include scattering processes involving the top quark Yukawa coupling, with rate $\Gamma_{y}$, and in the phase boundary and broken phase we have Higgs violating processes at a rate $\Gamma_{h}$ and axial top number violation at a rate $\Gamma_{m}$. Following ref. [5], particle transport is treated by including a diffusion term. We take all the quarks and squarks to have the same diffusion constant $D_{q}$ and the Higgs and Higgsinos to have diffusion constant $D_{h}$.

The rates of change of the various densities are now described by the coupled equations:

$$
\begin{aligned}
& \dot{Q}=D_{q} \nabla^{2} Q-\Gamma_{y}\left[Q / k_{Q}-H / k_{H}-T / k_{T}\right]-\Gamma_{m}\left[Q / k_{Q}-T / k_{T}\right] \\
& -6 \Gamma_{s s}\left[2 Q / k_{Q}-T / k_{T}+9(Q+T) / k_{B}\right]+\gamma_{\tilde{s}} \\
& \dot{T}=D_{q} \nabla^{2} T-\Gamma_{y}\left[-Q / k_{Q}+H / k_{H}+T / k_{T}\right] \\
& -\Gamma_{m}\left[-Q / k_{Q}+T / k_{T}\right]+3 \Gamma_{s s}\left[2 Q / k_{Q}-T / k_{T}+9(Q+T) / k_{B}\right]-\gamma_{\tilde{s}} \\
& \dot{H}=D_{h} \nabla^{2} H-\Gamma_{y}\left[-Q / k_{Q}+T / k_{T}+H / k_{H}\right]-\Gamma_{h} H / k_{H}+\gamma_{\tilde{h}} .
\end{aligned}
$$

Several simplifications of equations (3.5) can be made. First we ignore the curvature of the bubble wall, and so $\Gamma_{m}, \Gamma_{h}$, and $\gamma_{\tilde{s}, \tilde{h}}$ are only functions of $\bar{z} \equiv\left|\vec{r}+\vec{v}_{w} t\right|$, where $\vec{v}_{w}$ is the bubble wall velocity. We will assume that the density perturbations of interest are only functions of $\bar{z}$, the coordinate normal to the wall surface.

With these assumptions we arrive at the equations for $Q(\bar{z}), T(\bar{z})$, and $H(\bar{z})$ in the rest frame of the bubble wall: 


$$
\begin{aligned}
& 0=- v_{w} Q^{\prime}+D_{q} Q^{\prime \prime}-\Gamma_{y}\left[Q / k_{Q}-H / k_{H}-T / k_{T}\right]-\Gamma_{m}\left[Q / k_{Q}-T / k_{T}\right] \\
& \quad-6 \Gamma_{s s}\left[2 Q / k_{Q}-T / k_{T}+9(Q+T) / k_{B}\right]+\gamma_{\tilde{s}} \\
& 0=- v_{w} T^{\prime}+D_{q} T^{\prime \prime}+-\Gamma_{y}\left[-Q / k_{Q}+H / k_{H}+T / k_{T}\right] \\
& \quad-\Gamma_{m}\left[-Q / k_{Q}+T / k_{T}\right]+3 \Gamma_{s s}\left[2 Q / k_{Q}-T / k_{T}+9(Q+T) / k_{B}\right]-\gamma_{\tilde{s}} \\
& 0=-v_{w} H^{\prime}+D_{h} H^{\prime \prime}-\Gamma_{y}\left[-Q / k_{Q}+T / k_{T}+H / k_{H}\right]-\Gamma_{h} H / k_{H}+\gamma_{\tilde{h}} .
\end{aligned}
$$

We now assume that the rates $\Gamma_{y}$ and $\Gamma_{s s}$ are fast, and so $Q / k_{Q}-H / k_{H}-T / k_{T}=$ $\mathcal{O}\left(1 / \Gamma_{y}\right), 2 Q / k_{Q}-T / k_{T}+9(Q+T) / k_{B}=\mathcal{O}\left(1 / \Gamma_{s s}\right)$. We will check later whether this assumption is self consistent. We then take the linear combination of eqs. (3.6) which is independent of $\Gamma_{s s}, \Gamma_{y}$, and substitute

$$
\begin{aligned}
Q & =H\left(\frac{k_{Q}\left(9 k_{T}-k_{B}\right)}{k_{H}\left(k_{B}+9 k_{Q}+9 k_{T}\right)}\right)+\mathcal{O}\left(1 / \Gamma_{s s}, 1 / \Gamma_{y}\right) \\
T & =-H\left(\frac{k_{T}\left(2 k_{B}+9 k_{Q}\right)}{k_{H}\left(k_{B}+9 k_{Q}+9 k_{T}\right)}\right)+\mathcal{O}\left(1 / \Gamma_{s s}, 1 / \Gamma_{y}\right) .
\end{aligned}
$$

We then find that the Higgs density satisfies

$$
0=-v_{w} H^{\prime}+\bar{D} H^{\prime \prime}-\bar{\Gamma} H+\bar{\gamma}+\mathcal{O}\left(1 / \Gamma_{s s}, 1 / \Gamma_{y}\right)
$$

where $\bar{D}$ is an effective diffusion constant, $\bar{\Gamma}$ is an effective decay constant and $\bar{\gamma}$ is an effective source term, given by

$$
\begin{aligned}
\bar{D} & =\frac{D_{q}\left(9 k_{Q} k_{T}-2 k_{Q} k_{B}-2 k_{B} k_{T}\right)+D_{H} k_{H}\left(9 k_{Q}+9 k_{T}+k_{B}\right)}{9 k_{Q} k_{T}-2 k_{Q} k_{B}-2 k_{B} k_{T}+k_{H}\left(9 k_{Q}+9 k_{T}+k_{B}\right)} \\
\bar{\gamma} & =\left(\gamma_{\tilde{s}}+\gamma_{\tilde{h}}\right)\left(\frac{k_{H}\left(9 k_{Q}+9 k_{T}+k_{B}\right)}{9 k_{Q} k_{T}-2 k_{Q} k_{B}-2 k_{B} k_{T}+k_{H}\left(9 k_{Q}+9 k_{T}+k_{B}\right)}\right) \\
\bar{\Gamma} & =\left(\Gamma_{m}+\Gamma_{h}\right)\left(\frac{9 k_{Q}+9 k_{T}+k_{B}}{9 k_{Q} k_{T}-2 k_{Q} k_{B}-2 k_{B} k_{T}+k_{H}\left(9 k_{Q}+9 k_{T}+k_{B}\right)}\right) .
\end{aligned}
$$

In these equations, $\bar{\gamma}$ is the sum of the rate of generation of axial quark number and Higgs number inside the wall as given in (2.34) and $(2.20)$ while, $\bar{\Gamma}$ is the total rate of relaxation for those charges. We estimate the latter to be

$$
\left(\Gamma_{m}+\Gamma_{h}\right) \approx \frac{4 M_{W}^{2}(T, z)}{21 g^{2} T} \lambda_{t}^{2} \sin ^{2} \beta+\frac{M_{W}^{2}(T, z)}{35 g^{2} T} .
$$


Equation (3.8) is easily solved numerically for arbitrary shape of the source $\bar{\gamma}$ and decay term $\bar{\Gamma}$, however in order to qualitatively understand how the baryon number produced depends on the various parameters we will approximate the source as a step function of width $w$

$$
\begin{array}{ll}
\bar{\gamma}=\tilde{\gamma}, & w>\bar{z}>0 \\
\bar{\gamma}=0, \quad \bar{z}>w, \quad \bar{z}<0
\end{array}
$$

while for the decay terms we take

$$
\begin{array}{ll}
\bar{\Gamma}=\tilde{\Gamma}, & \bar{z}>0 \\
\bar{\Gamma}=0, & \bar{z}<0 .
\end{array}
$$

The effective diffusion constant is also spatially varying since the statistical factors $k_{i}$ depend on spatially varying particle masses and since the weak interaction cross sections depend on the Higgs vevs, however we will make the reasonable approximation that $\bar{D}$ is constant. An analytic solution to eq. (3.8), which satisfies the boundary conditions $H( \pm \infty)=0$ is now readily found; for $\bar{z}<0$ (the symmetric phase) this is

$$
H=\mathcal{A} e^{\bar{z} v_{w} / \bar{D}}
$$

with

$$
\mathcal{A}=\frac{4 \tilde{\gamma} \bar{D}\left(1-e^{-\left[\left(v_{w}+\sqrt{4 \bar{D} \tilde{\Gamma}+v_{w}^{2}}\right)(\bar{D})\right]}\right)}{\left(v_{w}+\sqrt{4 \bar{D} \tilde{\Gamma}+v_{w}^{2}}\right)^{2}}
$$

We will see that $\bar{D} \tilde{\Gamma} \gg v_{w}^{2}$ and so a good approximation to eq. (3.14) is

$$
\begin{aligned}
\mathcal{A} & \approx\left(\frac{\tilde{\gamma}}{\tilde{\Gamma}}\right)\left(1-e^{-2 w \sqrt{\frac{\tilde{\Gamma}}{D}}}\right) \\
& \approx k_{H}\left(\frac{\gamma_{\tilde{s}}+\gamma_{\tilde{h}}}{\Gamma_{m}+\Gamma_{y}}\right)\left(1-e^{-2 w \sqrt{\frac{\tilde{\Gamma}}{D}}}\right) .
\end{aligned}
$$

From the form of (3.13), we see that the $C P$ violating densities are non zero for a time $t=\bar{D} / v_{w}^{2}$, and so the assumptions about which rates are fast which were used to derive eq. (3.8) are valid provided $\bar{D} \Gamma_{s s} / v_{w}^{2}, \bar{D} \Gamma_{y} / v_{w}^{2} \gg 1, \bar{D} \Gamma_{w s} / v_{w}^{2} \ll 1$, and the scattering processes due to Yukawa couplings other than top are slow. 
To estimate $\bar{D}$ we take the Higgs diffusion constant $D_{h}$ to be comparable to the diffusion constant for left handed leptons, which was estimated in the MSM in Ref. 447 to be $110 / T$ and take $D_{q}$ from [47] to be $6 / T$. (These numbers will decrease slightly due to the supersymmetric particle content of the plasma-we ignore this effect as being small compared with other uncertainties in our calculation.) For the $k_{i}$ 's we assume that all the supersymmetric particles are heavy compared with $T$ except for the neutralinos and charginos and so

$$
k_{Q} \approx 6, \quad k_{T} \approx 3, \quad k_{B} \approx 3, \quad k_{H} \approx 12
$$

We then find the effective diffusion constant defined in eq. (3.9) is large,

$$
\bar{D} \approx 100 / T
$$

The large effective diffusion constant indicates that most of the transport of $C P$ violating quantum numbers is done by weakly interacting particles, i.e.the Higgs and Higgsinos, and since Yukawa interactions readily convert Higgs number into axial top number, transport of axial top number is surprisingly efficient.

For the scattering rate due to the top quark Yukawa coupling we estimate

$$
\Gamma_{y} \approx(27 / 2) \lambda_{t}^{2} \alpha_{s}\left(\zeta(3) / \pi^{2}\right)^{2} T
$$

and so $\bar{D} \Gamma_{y} / v_{w}^{2} \gtrsim 2 / v_{w}^{2}$ and the assumption that this rate is fast is self consistent. The next largest Yukawa coupling is the bottom quark's. Including scattering from this Yukawa coupling would give corrections to our results of order

$$
\sim \bar{D}\left((27 / 2) \lambda_{b}^{2} \alpha_{s}\left(\zeta(3) / \pi^{2}\right)^{2} T\right) / v_{w}^{2}
$$

We assume that the ratio $\tan \beta$ of Higgs expectation values is not unnaturally large, and so scattering due to the bottom and other Yukawa couplings may consistently be neglected for $v_{w} \gtrsim 10^{-2}$.

For the anomalous fermion number violating rates we take

$$
\Gamma_{w s}=6 \kappa \alpha_{w}^{4} T, \quad \Gamma_{s s}=6 \kappa^{\prime} \frac{8}{3} \alpha_{s}^{4} T
$$


where $\kappa, \kappa^{\prime}$ are unknown parameters usually assumed to be of order one. Thus the weak sphaleron rate may safely be taken to be slow provided

$$
\kappa / v_{w}^{2} \lesssim 10^{4}
$$

and the strong sphaleron rate is fast if

$$
\kappa^{\prime} / v_{w}^{2} \gtrsim 5
$$

In our computation of the baryon asymmetry we will approximate the strong sphaleron rate as fast and the weak sphaleron rate as slow.

What we set out to compute was not the Higgs density in the symmetric phase but the total baryon number density left inside the bubble. We now turn the weak sphaleron rate on, assuming it has a negligible effect on particle densities (eq. (3.21) is valid), however it provides the only source for net baryon number. We thus take $\rho_{B}$, the baryon number density, to be a function of $\bar{z}$ satisfying

$$
0=D_{q} \rho_{B}^{\prime \prime}-v_{w} \rho_{B}^{\prime}-\Theta(-\bar{z}) n_{F} \Gamma_{w s} n_{L}(\bar{z})
$$

where $n_{L}$ is the total number density of left handed weak doublet fermions, $n_{F}=3$ is the number of families, and we have assumed that anomalous baryon number creation takes place only for $\bar{z}<0$ (the symmetric phase). Eq. (3.23) has solution

$$
\rho_{B}(\bar{z})=-\frac{3 \Gamma_{w s}}{v_{w}} \int_{-\infty}^{0} n_{L}(\bar{z}) d \bar{z}-\frac{3 \Gamma_{w s}}{v_{w}} \int_{0}^{\bar{z}} d z^{\prime} \Theta\left(-z^{\prime}\right) n_{L}\left(z^{\prime}\right)\left(1-e^{v_{w}\left(\bar{z}-z^{\prime}\right) / D_{q}}\right),
$$

which is a constant for $\bar{z}>0$ and vanishes as $\bar{z} \rightarrow-\infty$. Thus, up to corrections of order $\Gamma_{w s} \bar{D} / v_{w}^{2}$, the baryon density inside the bubbles of broken phase is simply proportional to the integral of $n_{L}$ in the symmetric phase.

We now return to eq. (3.6), in order to compute $n_{L}$. As pointed out by Giudice and Shaposhnikov [46], if we use eq. (3.16) we will find the answer is zero in the limit $\Gamma_{s s} \rightarrow \infty$, so we need to compute the $\mathcal{O}\left(1 / \Gamma_{s s}\right)$ corrections to particle densities 13 . We will assume

13 In this limit, one should also include the contribution of sources for conserved charges: $B-L$, .... Local densities of conserved charges are also generated by the scattering of particles on the moving wall through charge separation. As discussed in section $\S 2.1$, these sources are subleading, however they do not suffer from the strong sphaleron suppression. 
$\Gamma_{y} \gg \Gamma_{s s},\left(\kappa^{\prime} \lesssim 7\right)$ and take

$$
\begin{aligned}
& Q=H\left(\frac{k_{Q}\left(9 k_{T}-k_{B}\right)}{k_{H}\left(k_{B}+9 k_{Q}+9 k_{T}\right)}\right)+\delta_{Q}+\mathcal{O}\left(1 / \Gamma_{y}\right) \\
& T=-H\left(\frac{k_{T}\left(2 k_{B}+9 k_{Q}\right)}{k_{H}\left(k_{B}+9 k_{Q}+9 k_{T}\right)}\right)+\left(\frac{k_{T}}{k_{Q}}\right) \delta_{Q}+\mathcal{O}\left(1 / \Gamma_{y}\right) .
\end{aligned}
$$

Substituting these values into eq. (3.6), we find

$$
\begin{aligned}
0= & D_{q}\left(Q^{\prime \prime}+T^{\prime \prime}\right)+v\left(Q^{\prime}+T^{\prime}\right)-3 \Gamma_{s s}\left(\frac{2 Q}{k_{Q}}-\frac{T}{k_{T}}+\frac{9(Q+T)}{k_{B}}\right) \\
= & \left(\frac{-k_{B}\left(k_{Q}+2 k_{T}\right)}{k_{H}\left(9 k_{Q}+9 k_{T}+k_{B}\right)}\right)\left(D_{q} H^{\prime \prime}-v_{w} H^{\prime}\right)-3 \Gamma_{s s}\left(\frac{k_{B}+9 k_{Q}+9 k_{T}}{k_{B} k_{Q}}\right) \delta_{Q} \\
& +\mathcal{O}\left(1 / \Gamma_{s s}, 1 / \Gamma_{y}\right) \\
\Rightarrow \delta_{Q}= & \left(\frac{D_{q} H^{\prime \prime}-v_{w} H^{\prime}}{\Gamma_{s s}}\right)\left(\frac{-k_{B}^{2} k_{Q}\left(k_{Q}+2 k_{T}\right)}{3 k_{H}\left(9 k_{Q}+9 k_{T}+k_{B}\right)^{2}}\right)+\mathcal{O}\left(1 / \Gamma_{s s}^{2}, 1 / \Gamma_{y}\right) .
\end{aligned}
$$

We now solve algebraically for $n_{L}=Q+Q_{1 L}+Q_{2 L}$ using eqs. (3.3), (3.25), and (3.26), and find

$$
\begin{aligned}
n_{L} & =5 Q+4 T \\
& =\left(\frac{5 k_{Q}+4 k_{T}}{k_{Q}}\right) \delta_{Q}+\left(\frac{9 k_{Q} k_{T}-8 k_{B} k_{T}-5 k_{B} k_{Q}}{k_{H}\left(k_{B}+9 k_{Q}+9 k_{T}\right)}\right) H .
\end{aligned}
$$

If we use eq. (3.16) we find

$$
n_{L}=7 \delta_{Q}=-1 / 56\left(\frac{D_{q} H^{\prime \prime}-v_{w} H^{\prime}}{\Gamma_{s s}}\right)
$$

so the baryon density is proportional to $\Gamma_{w s} / \Gamma_{s s}$, and is only sensitive to the ratio of $\kappa / \kappa^{\prime}$, provided eqs. (3.21) and (3.22) are satisfied. This reduces the uncertainty in the baryon asymmetry since estimates for both $\kappa$ and $\kappa^{\prime}$ vary by several orders of magnitude but we expect the ratio to be approximately one. The result that $n_{L}$ and the baryon density are suppressed by a factor of $1 / \Gamma_{s s}$ does not hold if one considers modifications to eq. (3.16) due to higher order corrections [46] or due to the contributions of nondegenerate squarks. The cancellation which makes the first term of eq. (3.27) dominate the second term no longer occurs when nondegenerate masses are considered. However examination of eqs. (3.26) and (3.27) shows that corrections from this lack of cancellation are negligible unless either $\kappa^{\prime} / v_{w}^{2} \gtrsim 10^{3}$, or some squarks are not much heavier than $T$. Note that there 
can be significant enhancement of the baryon density if, for example, only the top squark is light in the symmetric phase, as we will discuss at the end of this section. With all squarks heavy, our final answer for the baryon to entropy ratio in the broken phase, combining eqs. (3.13), (3.15), (3.24), (3.26), and (3.27) is

$$
\begin{aligned}
\frac{\rho_{B}}{s} & =-\left(\frac{3 \mathcal{A} \Gamma_{w s}}{56 s \Gamma_{s s}}\right)\left(1-\frac{D_{q}}{\bar{D}}\right) \\
& \approx-3.5 \times 10^{-5} \gamma_{w} v_{w}\left(\frac{\kappa}{\kappa^{\prime}}\right) \mathcal{S}
\end{aligned}
$$

with

$$
\mathcal{S}=\frac{\left(\gamma_{\tilde{s}}+\gamma_{\tilde{h}}\right) w}{\left(\Gamma_{m}+\Gamma_{h}\right) \gamma_{w} v_{w} T^{2}}\left(\frac{1-e^{-2 w \sqrt{\frac{\tilde{\Gamma}}{D}}}}{w T}\right)
$$

We have taken the entropy $s$ to be $s=\left(2 \pi^{2} g_{*} / 45\right) T^{3}=55.1 T^{3} \quad\left(g_{*}=1253 / 4\right)$ and we have made explicit the dependence on the velocity $v_{w}$ and the thickness of the wall $w$.

The factor $\mathcal{S}$ is a dimensionless number, function of the supersymmetric parameters $\mu, \sin \phi_{B}, \tilde{m}_{1,2}, A, \sin \phi_{A}, \tan \beta$ and $\Delta \beta$, the total variation of $\beta$ in the wall, as well as a function of the known gauge and top Yukawa couplings and $W$ and $Z$ masses. In short, $\mathcal{S}$ is a concise representation of the dependence of the baryon asymmetry produced on the yet unknown supersymmetric parameters of the SSM.

To compute the baryon asymmetry, we need to compute the factor in parenthesis in Eq. (3.30). This factor has its origin in the mechanism which transports the $C P$ violating asymmetries in front of the wall. If this transport is efficient, the answer should become independent of the wall thickness $w$. Indeed, using our estimates, 100/T, for $\tilde{D}$ given in (3.17) and our estimates for $\tilde{\Gamma}$ in (3.9) and $(3.10)$, we find $2 \sqrt{\tilde{\Gamma} / \tilde{D}} \simeq 5 \times$ $10^{-3} \sqrt{7 \sin ^{2} \beta+1} T$, a value fairly insensitive on the supersymmetric parameters. Hence, unless the wall thickness $w$ is anomalously large, 14 we find the factor in parenthesis to be equal to $5 \times 10^{-3} \sqrt{7 \sin ^{2} \beta+1}$ and, at leading order, independent of the wall thickness.

The $\mathcal{S}$-factor becomes, using general expressions for the source terms given in (2.20), (2.21) and (2.34), (2.35), and our expressions for the relaxation rates $\Gamma_{m}$ and $\Gamma_{h}$ in (3.10),

14 Typical estimates for $w T$ range between 10 and 100. 


$$
\begin{array}{r}
\mathcal{S} \approx 5.5 \times 10^{-3} \frac{\mu}{T} \frac{\tilde{m}_{2}}{T} \sin \phi_{B} \Delta \beta / \sqrt{7 \sin ^{2} \beta+1} \times\{ \\
\left(\mathcal{I}_{\tilde{h}}^{\mu}\left(1+\frac{\tilde{m}_{1}}{\tilde{m}_{2}} \frac{1}{10}\right)+\mathcal{I}_{\tilde{h}}^{\tilde{m}_{2}}+\mathcal{I}_{\tilde{h}}^{\tilde{m}_{1}} \frac{\tilde{m}_{1}}{\tilde{m}_{2}} \frac{1}{10}\right) \sin \phi_{B}+ \\
\left.\left(\left[\mathcal{I}_{\tilde{s}}^{\tilde{m}_{L}}+\mathcal{I}_{\tilde{s}}^{\tilde{m}_{R}}\right] 10 \frac{A}{\tilde{m}_{2}}\right) \sin \left(\phi_{B}-\phi_{A}\right)\right\}
\end{array}
$$

The first term in parenthesis represents the contribution of the charginos and neutralinos while the second term represents the contribution of the top squarks.

To try this formula, we use light neutralinos and charginos: $\mu=\tilde{m}_{2}=2 \tilde{m}_{1}=50$ $\mathrm{GeV}$, relatively heavy squarks; $\tilde{m}_{L, R}=150 \mathrm{GeV}$, and take $A=50 \mathrm{GeV}$. We need to know the ratio of these masses over the transition temperature $T$. The latter has its value completely determined by the parameters of the theory; in our analysis, however, it is a free parameter. As an indicative value, we choose $60 \mathrm{GeV}$ 罒. For these values, we find $\mathcal{I}_{\tilde{h}}^{\mu}=\mathcal{I}_{\tilde{h}}^{\tilde{m}_{2}} \approx 5.5 \times 10^{-2}, \mathcal{I}_{\tilde{h}}^{\tilde{m}_{1}} \approx 3.5 \times 10^{-2}$ and $\mathcal{I}_{\tilde{s}}^{\tilde{m}_{L, R}} \approx 2.4 \times 10^{-4}$. the $\mathcal{S}$-factor becomes

$$
\begin{aligned}
\mathcal{S} & \approx\left(\frac{5}{6}\right)^{2} \frac{\Delta \beta}{\sqrt{1+7 \sin ^{2} \beta}}\left(0.032 \sin \phi_{B}+5 \times 10^{-3} \sin \left(\phi_{B}-\phi_{A}\right)\right) 5.5 \times 10^{-3} \\
& \approx\left(1.8 \times 10^{-4} \sin \phi_{B}+6.6 \times 10^{-6} \sin \left(\phi_{B}-\phi_{A}\right)\right) \Delta \beta
\end{aligned}
$$

It is clear from the above equation that the squark contribution is only significant in the limit $\phi_{A} \gg \phi_{B}$ or in the limit the charginos and neutralinos are heavy.

Gathering all the above information, we find the following results. The largest contribution arises from light charginos and/or neutralinos, in which case, the asymmetry can be as large as

$$
\frac{\rho_{B}}{s} \approx-\gamma_{w} v_{w}\left(\frac{\kappa}{\kappa^{\prime}}\right) \sin \phi_{B} \Delta \beta 6.5 \times 10^{-9} .
$$

The measured baryon asymmetry is $(4-7) \times 10^{-11}$. So, electroweak baryogenesis is significant provided that

$$
\left(\frac{\kappa}{\kappa^{\prime}}\right)\left|\gamma_{w} v_{w} \sin \phi_{B} \Delta \beta\right| \geq 7.5 \times 10^{-3}
$$

15 Generically, one expects the temperature to be below the one in the SM $(\sim 80-100$ $\mathrm{GeV}$ ), as, in the MSM, the superpartners contribute to the effective potential in a manner which decreases the critical temperature. 
and is negative in sign. Let us discuss the magnitude of each term separately. $\kappa$ and $\kappa^{\prime}$ are two not well-known parameters characterizing the strength of the electroweak and strong anomalous processes, respectively, however, their ratio is expected to be of order one. In the minimal standard model, the wall velocity, $\gamma_{w} v_{w}$, is no smaller than 0.0216 and is more likely of order 0.117 or larger [24,43]; although no calculation has been done for SSM, it is reasonable to assume similar values. Finally, $\Delta \beta$ is the overall variation of the ratio of the two Higgs expectation values $v_{2}$ and $v_{1}$. As we argue in $\S 5$, its presence is an artefact of working at fourth order in the mass, it can be removed at the cost of introducing additional mass suppressions. From these considerations, we infer an optimal bound on the $C P$ violating phase $\phi_{B}$

$$
\left|\sin \phi_{B}\right| \geq 0.025
$$

Only with this bound satisfied, is electroweak baryogenesis achievable in SSM with light charginos and/or neutralinos and heavy and degenerate squarks.

In the case of neutralinos and charginos which are heavier than $T$ or $\phi_{A} \gg \phi_{B} \sim 0$, only top squarks contribute to the asymmetry. If all squarks are degenerate, they must all be heavier than $\sim 150 \mathrm{GeV}$ in which case, the requirement becomes

$$
\left|\Delta \beta \sin \left(\phi_{A}-\phi_{B}\right)\right| \geq 0.65
$$

which leads to experimentally ruled out electric dipole moments. Also, unlike the charginos/neutralinos case, $\Delta \beta$ must be non-zero and its presence is not an artefact of our approximations; $\Delta \beta$ can be significantly smaller than one. Clearly, electroweak baryogenesis in SSM with all neutralinos, charginos and squarks heavy is likely to be incompatible

16 This lower bound corresponds to the situation of maximal damping of the motion of the wall in the plasma, that is, it corresponds to the thin wall situation where mean free paths are larger than the thickness of the wall, $w$. This lower bound is a decreasing function of the Higgs and top quark masses; the specific value 0.02 has been computed following Ref. [24] for the values $m_{H} \sim 65 \mathrm{GeV}$ and $m_{t} \sim 175 \mathrm{GeV}$.

17 This larger value accounts for thermal scattering within the wall as, in the MSM, the wall thickness $w$ is typically larger than the mean free paths of the $W$ 's and $Z$ 's, $\tau_{w}$, and of the top quark, $\tau_{s}$. Large uncertainties arise from our imprecise knowledge of the ratios $\tau_{w, s} / w$. 
with constraints from electric dipole moments. We will discuss bounds (3.35) and (3.36) along with their uncertainties in the last section.

These conclusions are altered considerably if, say, the left handed bottom squark and left and right handed top squark masses squared are rather light, but the other squark masses are heavy. (This mass pattern could be a result of renormalization due to the large top Yukawa coupling near the Planck scale.) Then the factor multiplying $H$ in eq. (3.27) does not vanish. If we take

$$
k_{Q} \approx 18, \quad k_{T} \approx 9, \quad k_{B} \approx 3, \quad k_{H} \approx 12
$$

we have

$$
\begin{gathered}
n_{L}=\frac{27}{82} H, \\
\bar{D} \approx 72 / T,
\end{gathered}
$$

and

$$
\frac{\rho_{B}}{s}=-\left(\frac{81 \mathcal{A} \bar{D} \Gamma_{w s}}{82 v_{w}^{2} s}\right),
$$

i.e., $\rho_{B}$ is enhanced by a factor of $\sim 18 \bar{D} \Gamma_{s s} / v_{w}^{2}$ over the case with no light squarks and fast strong sphalerons, and is sensitive to the weak sphaleron rate rather than the ratio of weak and strong sphaleron rates. After a few substitutions, we obtain

$$
\frac{\rho_{B}}{s} \approx-1.5 \times 10^{-4} \mathcal{S} / v_{w}
$$

where we have made use of the $\mathcal{S}$-factor defined in (3.30) and (3.31). To obtain a numerical estimate, let us assume the values $A \simeq \mu \simeq \tilde{m}_{2} \simeq 2 \tilde{m}_{1} \simeq 50 \mathrm{GeV}$, and $\tilde{m}_{L, R} \simeq T \simeq 60$ $\mathrm{GeV}$. The factors in (3.31), are now $\mathcal{I}_{\tilde{h}}^{\mu}=\mathcal{I}_{\tilde{h}}^{\tilde{m}_{2}} \approx 5.5 \times 10^{-2}, \mathcal{I}_{\tilde{h}}^{\tilde{m}_{1}} \approx 3.5 \times 10^{-2}$ and $\mathcal{I}_{\tilde{s}}^{\tilde{m}_{L, R}} \approx 0.018$. the $\mathcal{S}$-factor becomes

$$
\begin{aligned}
\mathcal{S} & \approx\left(\frac{5}{6}\right)^{2} \frac{\Delta \beta}{\sqrt{1+7 \sin ^{2} \beta}}\left(0.032 \sin \phi_{B}+0.093 \sin \left(\phi_{B}-\phi_{A}\right)\right) 5.5 \times 10^{-3} \\
& \approx\left(1.8 \times 10^{-4} \sin \phi_{B}+5 \times 10^{-4} \sin \left(\phi_{B}-\phi_{A}\right)\right) \Delta \beta
\end{aligned}
$$

This time the contribution of the top squarks - the second term in parenthesis, is potentially as significant as the one of the light charginos or neutralinos. Combining this result with (3.41), we find 


$$
\frac{\rho_{B}}{s} \approx-\frac{\kappa}{v_{w}}\left(\Delta \beta \sin \phi_{B} 2.5 \times 10^{-8}+\Delta \beta \sin \left(\phi_{B}-\phi_{A}\right) 7.5 \times 10^{-8}\right)
$$

This is a significant contribution to the baryon-to-entropy ratio provided that

$$
\left|\Delta \beta \sin \phi_{B}+3 \Delta \beta \sin \left(\phi_{B}-\phi_{A}\right)\right| \geq \frac{v_{W}}{\kappa} 1.9 \times 10^{-3}
$$

and is negative in sign.

If we use the range $0.1-0.3$ for the wall velocity $v_{w}$ and the range $0.1-1$ for $\kappa$, and take masses for the superpartners which are optimal for baryogenesis, we obtain the following constraint on the magnitude of the phases $\phi_{A}$ and $\phi_{B}$

$\begin{array}{ccc}\text { light charginos/neutralinos/top squarks } & \left|\Delta \beta \sin \phi_{B}\right| & \geq 2 \times 10^{-4}-6 \times 10^{-3} \\ \text { light top squarks/charged higgs } & \left|\Delta \beta \sin \left(\phi_{B}-\phi_{A}\right)\right| & \geq 7 \times 10^{-5}-2 \times 10^{-3}\end{array}$

We emphasize that in the term contributed by the charginos and neutralinos, $\Delta \beta$ will not be present at higher order in the mass expansion. By taking the top squarks to be light we obtain a possible two-order of magnitude enhancement in $\rho_{B} / s$ over the situation with all squarks degenerate and heavier that $T$ (cf. (3.34)-(3.36)).

We also obtain qualitatively similar results to eq. (3.40) if strong sphalerons are slow, i.e. eq. (3.22) does not hold. Our formulae are also radically modified if weak sphalerons are sufficiently fast and/or if the wall velocities are so slow that eq. (3.21) is violated. Then most of our simplifications of the rate equations, such as the neglect of leptons, are invalid. We then expect the final answer for $\rho_{B}$ to be insensitive to the sphaleron rates, being determined by near-equilibrium physics.

\section{Baryon density in the Two Higgs model}

We can now easily solve for the baryon density in the two Higgs model since the particle transport equations are very similar to those in the SSM. Eq. (3.6) is unchanged, if we take the squark and Higgsino contributions to be zero, $\gamma_{\tilde{s}}$ to be the source for axial top number due to the top quark, $\gamma_{q}$ and we substitute $\gamma_{H}$, the source for Higgs number 
due to the Higgs particles, for $\gamma_{\tilde{h}}$. Finally, in the case of two(one) light Higgs, the statistical factors become

$$
k_{Q} \approx 6, \quad k_{T} \approx k_{B} \approx 3, \quad k_{H} \approx 8(4)
$$

and the effective diffusion constant, from (3.9)

$$
\bar{D} \approx \frac{96}{T}\left(\frac{88}{T}\right) .
$$

In Ref. [8] we computed $\gamma_{q}$ to be

$$
\gamma_{q}(\vec{x}, t) \simeq-\frac{N_{c}}{2 \pi^{2}} \gamma_{w} v_{w} T\left|m_{t}\right|^{2} \partial_{z} \theta+\mathcal{O}\left(v_{w}^{2},(\tau / w)^{2}\right)
$$

where $m_{t}(z),=\left|m_{t}(z)\right| e^{i \theta(z)}$, is the space-dependent mass of the top quark expressed in the wall frame. To find $\gamma_{H}$, we need to track the evolution of the Higgs number carried by the Higgses $H_{1}$ and $H_{2}$ as they evolve in the background of the wall. The space-dependent mass matrix is, in the basis $H_{1}, H_{1}^{*}, H_{2}$ and $H_{2}^{*}$,

$$
\mathcal{M}_{\tilde{H}}=\left(\begin{array}{cccc}
\ldots & m_{11}^{2} e^{-i \theta_{11}} & \ldots & m_{21}^{2} e^{-i \theta_{21}} \\
m_{11}^{2} e^{i \theta_{11}} & \cdots & m_{12}^{2} e^{i \theta_{12}} & \cdots \\
\ldots & m_{12}^{2} e^{-i \theta_{12}} & \ldots & m_{22}^{2} e^{-i \theta_{22}} \\
m_{21}^{2} e^{i \theta_{21}} & \ldots & m_{22}^{2} e^{i \theta_{22}} & \cdots
\end{array}\right) .
$$

We only displayed entries which violate Higgs number as they are the ones which control the charge generation as Higgs particles flow across the wall. The Higgs number charge operator takes the form

$$
\hat{Q}_{H}=\operatorname{Diag}(1,-1,1,-1)
$$

The analysis follows the steps of the one of the stop axial charge generation; in particular, Eq. (2.29) and (2.30) are directly transposable. We obtain

$$
\gamma_{H}(\vec{x}, t)=\gamma_{w} v_{w} \frac{1}{2 \pi^{2}} \times\left(\mathcal{J}_{H}^{m_{1}} \mathcal{I}_{H}^{m_{1}}+\mathcal{J}_{H}^{m_{2}} \mathcal{I}_{H}^{m_{2}}\right)+\mathcal{O}\left(v_{w}^{2},(\tau / w)^{2}\right)
$$

with

$$
\begin{aligned}
& \mathcal{J}_{H}^{m_{1}}=2 \partial_{z} \theta_{11} m_{11}^{4} / T+\partial_{z} \theta_{21} m_{21}^{4} / T+\partial_{z} \theta_{12} m_{12}^{4} / T \\
& \mathcal{J}_{H}^{m_{2}}=2 \partial_{z} \theta_{22} m_{22}^{4} / T+\partial_{z} \theta_{21} m_{21}^{4} / T+\partial_{z} \theta_{12} m_{12}^{4} / T .
\end{aligned}
$$

The function $\mathcal{I}_{H}^{m_{i}}$ is identical to the one computed for the squark, $\mathcal{I}_{\tilde{s}}^{m}$ in Eq. (2.35). The damping rate is set by weak interactions, our estimate is $\tau_{H} \sim 25 / T$. We choose for the 
on-shell masses $m_{1}$ and $m_{2}$ of the propagating Higgses the zero-momentum contributions that the Higgs particles receive from plasma interactions in both phases: $m_{1} \sim m_{2} \sim T / 3$ [36].

With these values, $\mathcal{I}_{H}^{m_{i}} \approx 0.25$ and, within our approximations,

$$
\begin{aligned}
\gamma_{H}(\vec{x}, t) \simeq & \gamma_{w} v_{w} \frac{1}{4 \pi^{2}} \times \\
& \left\{\partial_{z} \theta_{11} m_{11}^{4} / T+\partial_{z} \theta_{22} m_{22}^{4} / T+\partial_{z} \theta_{21} m_{21}^{4} / T+\partial_{z} \theta_{12} m_{12}^{4} / T\right\} .
\end{aligned}
$$

Combining eqs. (3.13), (3.15), (3.24), (3.26), and (3.27), the baryon to entropy ratio is

$$
\begin{aligned}
\frac{\rho_{B}}{s} & =-\left(\frac{3 \mathcal{A} \Gamma_{w s}}{56 s \Gamma_{s s}}\right)\left(1-\frac{D_{q}}{\bar{D}}\right) \\
& \approx-2.4 \times 10^{-5} \gamma_{w} v_{w}\left(\frac{\kappa}{\kappa^{\prime}}\right) \mathcal{H}
\end{aligned}
$$

with most of the parameter dependence contained in the $\mathcal{H}$ factor

$$
\mathcal{H}=\frac{\left(\gamma_{q}+\gamma_{h}\right) w}{\left(\Gamma_{m}+\Gamma_{h}\right) \gamma_{w} v_{w} T^{2}}\left(\frac{1-e^{-2 w \sqrt{\frac{\tilde{\Gamma}}{D}}}}{w T}\right)
$$

From (3.9), we compute $\bar{\Gamma}=0.11\left(\Gamma_{m}+\Gamma_{h}\right)$. We estimate $\Gamma_{m} \approx \lambda_{t}^{2} T / 21$ and we parametrize $\Gamma_{h} \approx \lambda^{2} T / 140$ with $\lambda$, an undefined parameter function of the Higgs quartic couplings. As in the SSM, charges diffuse a long distance in front of the wall $\bar{D} \gg \bar{\Gamma}$ and the term in parenthesis in Eq. (4.10), is largely independent of the wall thickness $w$. We find

$$
\mathcal{H}=\frac{\left(\gamma_{q}+\gamma_{h}\right) w}{\sqrt{\left(\Gamma_{m}+\Gamma_{h}\right) / T} \gamma_{w} v_{w} T^{3}} 7 \times 10^{-3} .
$$

Without going into a difficult study of the vast parameter space of the two Higgs models, we can obtain a fair estimate of the above quantity by neglecting the Higgs contributions to the source and to the rate, for the following reasons. The source $\gamma_{H}$ written in (4.8), is a linear combination of terms of the form $m_{i j}^{4} / T \partial_{z} \theta_{i j}$. These terms all violate Higgs number, hence, they are proportional to the quartic self-couplings $\sim \lambda^{2}\left(\Delta \theta_{i j} / w\right) T^{4}$ and are smaller than the contribution from the top quark $\sim \lambda_{t}^{2}\left(\lambda \Delta \theta_{i j} / w\right) T^{4}$, unless the Higgs sector is strongly coupled. Similarly, we expect $\Gamma_{m}>\Gamma_{h}$. Under these assumptions,

$$
\mathcal{H} \approx-10^{-2} \Delta \theta
$$


and

$$
\frac{\rho_{B}}{s} \approx \gamma_{w} v_{w}\left(\frac{\kappa}{\kappa^{\prime}}\right) \Delta \theta 2.5 \times 10^{-7}
$$

Choosing the illustrative value $\gamma_{w} v_{w} \sim 0.3$, this baryon per entropy ratio is significant provided that

$$
\left(\frac{\kappa}{\kappa^{\prime}}\right) \Delta \theta \geq 7 \times 10^{-4}
$$

\section{Outlook}

\subsection{Accuracy of present computations of the baryon asymmetry}

We now look back on the many approximations and uncertainties present in our analysis.

\section{- Approximations -}

1. For the purpose of solving Majorana, Dirac and Klein-Gordon equations, we performed an expansion in powers of $\mathcal{M}(x, t)$. The benefit was to work analytically and to express the answer as a sum of $C P$ violating invariants. The convergence of this expansion has been discussed and established in the discussion following Eq. (2.24). We have further approximated the density matrices describing particle distributions with Bose-Einstein and Fermi-Dirac distributions for on-mass shell particles in the unbroken phase. In ignoring the non-equilibrium component of the distribution, we are ignoring corrections of order $v_{w}^{2}$ (cf. discussion following Eq. (2.13)). In assuming on-mass shell particles in the unbroken phase, we are ignoring corrections of order $(m / T)^{2}$ and $(g v / T)^{2}$. Some particles, such as the squarks, are expected to have $S U(2) \times U(1)$ symmetric contributions to their masses which may be larger than the critical temperature $\left(T_{c} \sim 50-100 \mathrm{GeV}\right)$. However, as discussed in $\S 5.2$, heavy particles do not contribute significantly to baryogenesis. So, at best, we expect that accounting for the full mass dependence yields numerical corrections of order one. One exception is that for the neutralino and chargino contribution, when we work to lowest nontrivial order in the masses we obtain a result proportional to $\Delta \beta$-the change during the transition in the angle specifying the ratio of the Higgs vevs. There 
is no reason to expect this suppression factor to persist at higher orders in a mass expansion.

2. We defined our sources $\gamma_{Q}$ in a layer of a size $\tau$, the coherence time. To postpone recourse to numerical methods, we assumed $\tau$ to be smaller than the wall thickness $w$ and ignored corrections of order $(\tau / w)^{2}$. This is a very good approximation for strongly interacting particles but not necessarily for weakly interacting particles for which $\tau$ is in the range $(20-30) / T$ while the wall thickness $w$ can span the interval $(10-100) / T$. Only a precise calculation of these two quantities can decide the quality of this approximation. The largest $(\tau / w)^{2}$ corrections are contained in the factors $\mathcal{I}_{\tilde{h}, \tilde{s}}$. As explained in $\S 2$, we expect these factors, which at most increase linearly with $\tau$, to "saturate" for $\tau \gg w$, at about their values at $\tau \simeq w$. For this reason, we do not expect higher order terms to bring large corrections to our analysis.

3. We have made a number of simplifications of the equations describing particle transport and number changing processes. First, we assumed that deviations from thermal equilibrium were sufficiently small to allow us to describe particle distributions in terms of local chemical potentials and to make a diffusion approximation to transport processes. We expect this assumption to be quite good in the weakly interacting models considered. We simplified our treatment of diffusion in neglecting the finiteness of the speed of sound, that is, we worked at leading order in an expansion in $v_{w} / c_{s}$ where, $c_{s}=1 / \sqrt{3}$. Our choice of the magnitude of the wall velocity is such that it is a fair approximation. Should the wall velocity approach or be larger than the speed of sound, diffusion is not a good approximation to transport and our computations are invalid. An improved calculation which covers large wall velocities has yet to be developed. We made a severe approximation by simplifying the wall shape (eqs. (3.11) and $(3.12)$, which we expect to give an $\mathcal{O}(1)$ estimate of the true solution. We also made assumptions about the approximate rates of strong and weak sphalerons and the wall velocity, (eqs. (3.21) and (3.22)), and simplified our equations by assuming that the interactions proportional to the top Yukawa coupling were in thermal equilibrium. The size of corrections from these assumptions depends on how well the inequalities (3.21) and (3.22) are satisfied. We also assumed similar diffusion constants for all 
quarks, an error of order $\alpha_{w}^{2} / \alpha_{s}^{2} \sim 10 \%$. In fact this approximation for the quark diffusion constants is of very small numerical significance since diffusion is actually dominated by the weakly interacting Higgs, which provides a local source for axial top number far from the bubble wall. We also gave approximate estimates for the statistical factors $k_{i}$ defined by eq. (3.1)-here we expect corrections of order a few percent for light particles. The corrections to the quark statistical factors are important if $\kappa^{\prime} / v_{w}^{2} \gg \mathcal{O}\left(10^{2}\right)$ since from eqs. (3.24), (3.27) and (3.28) we see that they give the only contribution to the baryon asymmetry which is not suppressed by the strong sphaleron rate.

4. In most cases, our $\mathrm{CP}$ violating particle sources are dominated by the large $\mathrm{CP}$ violation in the transmission of low momentum particles over a distance $\Delta$ whose wavelength is comparable to $\Delta$. For these particles, kinetic theory starts to break down, giving corrections of $\mathcal{O}(1)$ to our treatment.

5. We have neglected the effects of long range gauge fields, which in general have an $\mathcal{O}(1)$ effect on the baryon density [39 41].

6. We have not included the contributions of the transport of conserved charges (such as B-L) to the baryon asymmetry. Such effects are higher order in wall velocity and masses, but may not suffer from the strong sphaleron suppression. We expect inclusion of such effects to change our results by at most $\mathcal{O}(1)$.

- Uncertainties -

Uncertainties in our estimate of the baryon asymmetry reflect not only the approximations above but also, and dominantly, our poor knowledge of certain parameters. Those are the coherence times $\tau_{\tilde{s}, \tilde{h}}$ and $\tau_{H, q}$, the diffusion constants $D$, the reaction rates $\Gamma_{y, h, m}$ and the parameters $\kappa$ and $\kappa^{\prime}$ measuring the strength of the anomalous processes. Fortunately in the most interesting situations, the latter occur in ratio which significantly decreases the uncertainty in the baryon asymmetry. Also, for large $\kappa$ the baryon asymmetry becomes insensitive to $\kappa$. Much work is needed to refine the determination of these parameters. Only $\tau, \kappa$ and $\kappa^{\prime}$ require understanding new physics; the determination of the other parameters faces only technical challenges. The parameters $v_{w}$ and $w$ describing the phase transition 
are also left free, both because they are parameter dependent and because of the lack of accurate computations for the models under consideration.

Finally we come to the main uncertainty, which is our lack of knowledge of the correct model of weak symmetry breaking and of the many new parameters introduced by any extension of the MSM. It is our hope that computation of the baryon asymmetry can provide a useful constraint on the weak symmetry breaking sector and on $C P$-violation.

Because of the above uncertainties and also because of the approximations that we described earlier, we believe that the computability of the baryon asymmetry produced is reliable to an order of magnitude. It is with this caveat that we now present our conclusions.

\subsection{Can the baryon asymmetry be produced in the SSM?}

Previous work on baryogenesis in supersymmetric models neglected the enhancing effects of transport, and concluded that sufficient $C P$-violation for baryogenesis in supersymmetric models could be marginally consistent with electric dipole moment constraints if one made optimistic assumptions about baryon number violating rates in the phase boundary [2,3], and if chargino and neutralino masses were not too heavy. Our work shows that with reasonable assumptions about the rates of anomalous processes, sufficient baryon asymmetry can be produced with small $C P$ violating phases of order $10^{-(2-4)}$, provided that the top squarks and either the neutralinos or the charginos are light compared with the transition temperature. If only the top squarks are light, it is also required that the ratio of Higgs vevs is not fixed during the transition, while when the inos are light, the lowest order contribution in $m / T$ is suppressed unless the ratio of Higgs vevs changes during the transition. The latter requirement implies that the effective theory during the transition has more than one light Higgs, which in turn means that at zero temperature the pseudoscalar and charged Higgs masses are not extremely heavy compared with the lightest Higgs mass. A light charged Higgs makes a potentially ruled out contribution to $b \rightarrow s \gamma$ [48] unless partially cancelled by a contribution from a loop containing light charginos and stops. We conclude that as far as sufficient $C P$ asymmetry is concerned, the SSM with some light superpartners $(\lesssim 100 \mathrm{GeV}$ ) is a good candidate for baryogenesis. With light

superpartners and with $C P$-violating phases of order $10^{-(2-4)}$, neutron and atomic electric 
dipole moments will be below the current experimental bounds [33, 35]. Furthermore, a large fraction of the relevant range of masses for the superpartners coincides with the range to be probed by LEP II.

In a calculation assuming only one light Higgs, the MSSM, with minimal superpartner content, has been shown to produce a phase transition sufficiently strongly first order to preserve the baryon asymmetry only when the lightest Higgs boson mass is less than 70 $\mathrm{GeV}$ and when at least some of top squarks are lighter than $110 \mathrm{GeV}$ [36]. We do not expect these bounds to be weakened significantly when the full parameter space for the Higgs masses is considered. Thus the baryon number washout constraint on the MSSM seems more powerful than the constraint of sufficient $C P$-violation. It is however subject to the uncertainties in the perturbative calculations of phase transition parameters.

\subsection{Conclusions about the baryon asymmetry in the two Higgs model, and comparison} with other calculations

To summarize $\S 4$, sufficient baryon asymmetry may easily be produced in a general model with two Higgs doublets and soft $C P$-violation in the scalar potential, with $C P$ violating phase as small as $\sim 7 \times 10^{-4}$ (eq. (4.14)). This result allows for a much smaller phase than most earlier calculations in the two Higgs model. Here we explain how our calculation differs the earlier ones.

The baryogenesis mechanism of axially asymmetric top quark reflection from the bubble walls [16] also allowed a small phase of order $10^{-5}$ in the two Higgs model, but only for the fine-tuned case where the bubble walls were thin, of order the inverse top mass. Refs. [16,31] concluded that when the walls are thick, a completely negligible $C P$-violating asymmetry is produced in the symmetric phase from top quark reflection. However in those papers several significant effects are neglected, such as thermal scattering within the phase boundary which is especially important for thick walls. Thermal scattering processes tend to interfere with baryogenesis by destroying the quantum coherence necessary for $C P$-violation [7,9], but also can in some cases enhance the baryon asymmetry produced. The enhancement comes about because $C P$-violating charge expectation values within the bubble wall can be converted to $C P$-violating thermal particle distributions inside the wall 
by incoherent thermal scattering processes, and these $C P$-violating thermal particle distributions can then diffuse into the symmetric phase, where they bias the relatively rapid anomalous weak processes towards producing net baryon number. We therefore find that the huge suppression of the top quark contribution to baryogenesis, found in refs. [16, 31] when the bubble walls are thick, is absent when thermal scattering and transport processes are considered. Instead, we find that the baryon asymmetry is not very sensitive to the width of the boundary.

Let us now compare our method of computation to two alternative methods which have appeared in the literature. For the case of thick boundaries an alternative method of calculation of the particle distributions in the wall, which should be about as accurate as the thick wall approximations we made, would be to use the method of linear response [18,49], i.e. to compute the charge current density produced from an initial $C P$ symmetric thermal particle distribution when space-time dependent $C P$ violating terms in the Hamiltonian are turned on for a time equal to the thermalization time $\tau$, and then dividing by $\tau$ to get the rate for production of a $C P$-violating charge in the phase boundary. Such a calculation can be done diagrammatically, e.g.by computing the diagrams considered in ref [50] (which however does not contain a linear response calculation, as in that work the effects of a finite $\tau$ are neglected). If one considers times longer than $\tau$ in the linear response, including the damping terms in the quark propagators which are generated by gluon exchange is essential. Another method of calculation has been developed in Ref. [51]. It consists of writing a Boltzmann equation for a one-particle distribution function which incorporates a $C P$-violating force term arising from the $C P$-violating space-dependent background. This equation is then solved for the resulting departure from thermal particle distributions, which is asymmetric between particles and their CP conjugates, and which are described in terms of chemical potentials. The latter are, in turn, inserted in a rate equation to compute a baryon asymmetry. It is not clear to us how to generalize this method to cover the case where several species mix, as occurs with the neutralinos and higgsinos in the SSM. In any case this method is semiclassical in nature and only describes the regime of $\tau$ much larger than particle wavelengths $(T \tau \gg 1)$. In this regime our calculations also produce a semi-classical fall-off of the $C P$ violating sources (cf. Fig. 3 and Fig. 5), 
in qualitative agreement with the analysis of ref [51 18. This calculation is appropriate for weakly interacting particles such as the $\tau$-lepton in the two Higgs model, but not for strongly interacting particles whose mean free paths are not much longer than their wavelengths and for which decoherence effects are already perceptible.

It is now evident that transport processes, omitted in the original thick wall calculations [2, 17, 19], significantly enhance the baryon asymmetry produced during the weak transition. In fact it has been suggested that the $\tau$-lepton plays a leading role in baryogenesis due to its large diffusion constant [42, 31]. However axial top quark number is also efficiently transported, because the large top Yukawa coupling allows axial top number to convert to Higgs number, which is transported by weakly interacting Higgs particles. Another argument in favor of the tau lepton contribution to baryogenesis dominating that of the top quark is that the the axial top number tends to be washed out by strong sphaleron processes. In fact we find that this suppression factor is only about $\sim 1 / 50$ for $\kappa^{\prime}$ of order one and wall velocities $v_{w} \sim 0.3$. Furthermore, even for arbitrarily fast strong sphaleron rate, the strong sphaleron suppression will never be more than about $10^{-3}$, due to the nondegenerate thermal masses of the quarks [46]. Despite the suppression factors for the top quark contribution, we believe the tau is likely to be less important than the top for baryogenesis in two Higgs models, because the source for axial tau number is suppressed relative to the axial top source by a factor of $\lambda_{\tau}^{2} / \lambda_{t}^{2}$, which is about $10^{-4}$ unless $\tan \beta$ is large. In the SSM, it is only possible to avoid having sphalerons wash out the baryon number if $\tan \beta$ is relatively small [36], and so there is no significant effect from the tau or scalar tau.

\section{Acknowledgements}

This work was supported in part by the DOE under contract \#DE-FG06-91-ER40614. The work of A. N. was supported in part by a fellowship from the Sloan Foundation. We gratefully acknowledge useful conversations and correspondance with G. Bonini, M.B. Gavela, M. Joyce and M.E. Shaposhnikov.

18 These authors coined the word "classical baryogenesis" to describe their analysis. However their "classical force" is not completely classical. That is, the spin dependent $C P$-violating term in their one particle Hamiltonian is $\propto \hbar \partial_{z} \theta$, where $\theta$ is the argument of the top quark mass. 


\section{References}

[1] V.A. Kuzmin, V.A. Rubakov, M.E. Shaposhnikov, Phys. Lett. 155B (1985) 36

[2] M. Dine, P. Huet, R. Singleton, Jr. and L. Susskind, Phys. Lett. B 257 (1991) 351.

[3] A. Cohen and A. Nelson, Phys. Lett. B297 (1992) 111.

[4] M. Joyce, T. Prokopec and N. Turok, preprint PUPT-1436 (1994), hep-ph/9401351

[5] A. G. Cohen, D. B. Kaplan and A. E. Nelson, Phys.Lett. B336 (1994) 41, hep$\mathrm{ph} / 9406345$

[6] D. Comelli, M. Pietroni and A. Riotto, preprint DFPD-94-TH-39, hep-ph/9406369

[7] P. Huet, E. Sather, hep-ph/9404302, Phys. Rev. D51 (1995) 379

[8] P. Huet and A.E. Nelson, Phys. Lett. B 355 (1995) 229, hep-ph/9504427

[9] M.B. Gavela, P. Hernandez, J. Orloff, O. Pene, C. Quimbay, Mod. Phys. Lett. A, Vol. 9, No. 9 (1994) 795; hep-ph/9406289, Nucl. Phys. (1994) B430 382

[10] G. 't Hooft, Phys. Rev. Lett. 37 (1976) 8

[11] P. Arnold and L. McLerran, Phys. Rev. D36 (1987) 581;Phys. Rev. D37 (1988) 1020

[12] J. Ambjorn, T. Askgaard, H. Porter, M. Shaposhnikov, Phys. Lett. 244B (1990) 479,Nucl. Phys. B353 (1990) 346

[13] J. Ambjorn and A. Krasnitz, preprint NBI-HE-95-23 (1995), hep-ph/9508202

[14] V. A. Kuzmin, V. A. Rubakov, M. E. Shaposhnikov, Phys. Lett. 191B (1987) 171

[15] A.G. Cohen, D.B. Kaplan, A.E. Nelson, Phys. Lett. 245B (1990) 561; Nucl. Phys. B349 (1991) 727

[16] A.E. Nelson, D.B. Kaplan, A.G. Cohen, Nucl. Phys. B373 (1992) 453

[17] L. McLerran, M. E. Shaposhnikov, N. Turok and M. Voloshin, Phys. Lett. B 256 (1991) 451.

[18] M. Dine, P. Huet and R. Singleton, Jr, Nucl.Phys. B375 (1992) 625.

[19] A.G. Cohen, D.B. Kaplan, A.E. Nelson Phys. Lett. B263 (1991) 86.

[20] A. G. Cohen, D. B. Kaplan, A. E. Nelson, hep-ph/9302210, Annu. Rev. Part. Nucl. Sci. 43 (1993) 27; P. Huet, hep-ph/9406301, Contributed to the First International Conference on Phenomenology of Unification from Present to Future, Roma, ITALY March 1994, SLAC-PUB-6492; D.B. Kaplan, hep-ph/9503360, Talk presented at "Beyond the Standard Model IV", Tahoe City 12/94, University of Washington Institute for Nuclear Theory preprint INT95-00-86

[21] M.E. Shaposhnikov, JETP Lett. 44 (1986) 364

[22] F. Klinkhamer and N. Manton, Phys. Rev. 30 (1984) 2212

[23] A.I. Bochkarev, M.E. Shaposhnikov, Mod. Phys. Lett. A2 (1987) 417

[24] M. Dine, P. Huet, R. G. Leigh, A. Linde and D. Linde, Phys. Rev. D 46 (1992) 550 .

[25] K. Farakos, K. Kajantie, K. Rummukainen, M. Shaposhnikov, hep-ph/9405234, Phys. Lett. B336 (1994) 494;1) Z. Fodor, J. Hein, K. Jansen, A. Jaster, I. Montvay, F. 
Csikor, hep-lat/9405021, Phys. Lett. B334 (1994) 405; hep-lat/9409017, Nucl. Phys. B439 (1995) 147

[26] P. Arnold, S. Vokos, Phys. Rev. 44 (1991) 3620; J.R. Espinosa, M. Quiros, hepph/9504241, Cern preprint CERN-TH-95-18 (1995)

[27] Glennys R. Farrar, M.E. Shaposhnikov, hep-ph/9305274, Phys. Rev. Lett. 70 (1993) 2833, ERRATUM-ibid.71:210,1993; hep-ph/9305275

[28] N. Turok, J. Zadrozny, Nucl. Phys. B358 (1991) 471.

[29] see e.g. S.M. Barr, Int. J. Mod. Phys. A8 (1993) 209 for a review

[30] D. Land, E. D. Carlson, Phys. Lett. B292 (1992) 107, hep-ph/9208227, A. Hammerschmitt, J. Kripfganz, M.G. Schmidt, Z. Phys. C64 (1994) 105

[31] J. M. Cline, K. Kainulainen, A. P. Vischer, preprint MCGILL-95-16, hep-ph/9506284

[32] J. Ellis, S. Ferrara, D.V. Nanopoulos, Phys. Lett. 114B (1982) 231; J. Polchinski and M. B. Wise, Phys. Lett. 125B (1983) 393; F. del Aguila, M.B. Gavela, J.A. Grifols, A. Mendez, Phys. Lett. 126B (1983) 71, ERRATUM-ibid.129B (1983) 473; M. Dugan, B. Grinstein, L. Hall, Nucl. Phys. B255 (1985) 413

[33] Y. Kizukuri and N. Oshimo, Phys. Rev. D45 (1992) 1806; Phys. Rev. D46 (1992) 3025

[34] S. Dimopoulos and L. J. Hall, Phys. Lett. B344 (1995) 185, hep-ph/9411273

[35] W. Fischler, S. Paban and S. Thomas (Texas U.) Phys. Lett. B289 (1992) 373, hep$\mathrm{ph} / 9205233$.

[36] J.R. Espinosa, M. Quiros and F. Zwirner, Phys. Lett. B307 (1993) 106, hepph/9303317; A. Brignole, J.R. Espinosa, M. Quiros and F. Zwirner, Phys. Lett. B324 (1994) 181, hep-ph/9312296

[37] M. E. Shaposhnikov, Phys. Lett. B277 (1992) 324, ERRATUM-ibid.B282:483,1992

[38] e.g. see J. F. Gunion, H. E. Haber, G. L. Kane and Sally Dawson preprint SCIPP89/13, (1989), erratum SCIPP-92-58, and refs therein

[39] S.Yu. Khlebnikov, Phys. Lett. 300B (1993) 376

[40] A.G. Cohen, D.B. Kaplan, A.E. Nelson, Phys. Lett. 294B (1992) 57

[41] J. M. Cline and K. Kainulainen preprint MCGILL-95-33, hep-ph/9506285

[42] M. Joyce, T. Prokopec and N. Turok, Phys. Lett. B338 (1994) 269, hep-ph/9401352

[43] B.-H. Liu, L. McLerran, N. Turok, Phys. Rev. D 46 (1992) 2668.

[44] E. Braaten and R. D. Pisarski, Phys. Rev. D 46 (1992) 1829; see also F. Flechsig, A. K. Rebhan, H. Schulz, hep-ph/9502324, DESY 95-022, ITP-UH-06/95 and references therein.

[45] Rabindra N. Mohapatra, Xin-min Zhang, Phys .Rev. D45 (1992) 2699

[46] G. Giudice and M. Shaposhnikov, Phys. Lett. 326B (1994) 118

[47] M. Joyce, T. Prokopec, N. Turok, PUPT-1495 (1994) hep-ph/9410281

[48] A.J. Buras and S. Pokorski, Nucl. Phys. B424 (1994) 374, hep-ph/9311345 
[49] E.M. Lifschitz and L.P. Pitaevkii, Statistical Physics, part 2 Pergamon Press, Oxford (1980); A.L. Fetter and J.D. Walecka, Quantum Theory of Many Particle Systems, McGraw Hill, New York (1971)

[50] D. Comelli, M. Pietroni and A. Riotto, preprint DESY-95-109, hep-ph/9506278

[51] M. Joyce, T. Prokopec and N. Turok, Phys.Rev.Lett. 75 (1995) 1695, hep-ph/9408339; preprint PUPT-1495, hep-ph/9410281; preprint PUPT-1496, hep-ph/9410282. 


\section{Figure Captions}

Fig. 1. (a) Amplitudes contributing to $J_{+}$. (b) Amplitudes contributing to $J_{-}$.

Fig. 2. (a) Contributions, to order $(\mathcal{M} / T)^{4}$, to the transmission amplitude $T$ of the neutralinos and the charginos. (b) Corresponding contributions, to order $(\mathcal{M} / T)^{3}$, to the reflection amplitude $R$.

Fig. 3. (a) The factor $\mathcal{I}_{\tilde{h}}^{m}$ plotted versus $\tau T$. $\mathcal{I}_{\tilde{h}}^{m}$ contains kinematic information on the propagation of the neutralinos and charginos in the plasma. (b) Its dependence on the mass eigenvalue $m$. The dots are the result from numerical integration and the solid lines are the fit (2.24).

Fig. 4. (a) Selected contributions, to order $(\mathcal{M} / T)^{4}$, to the transmission amplitude $T$ of the squarks. (b) Leading contributions, to order $(\mathcal{M} / T)^{2}$, to their reflection amplitude $R$.

Fig. 5. (a) The factor $\mathcal{I}_{\tilde{s}}^{m}$ plotted versus $\tau T$. This factor contains kinematic information on the propagation of the squarks in the SSM and on the propagation of the Higgs particles in two Higgs models. (b) Its dependence on the mass eigenvalue $m$. The dots are the result from numerical integration and the solid line is the fit (2.38). 

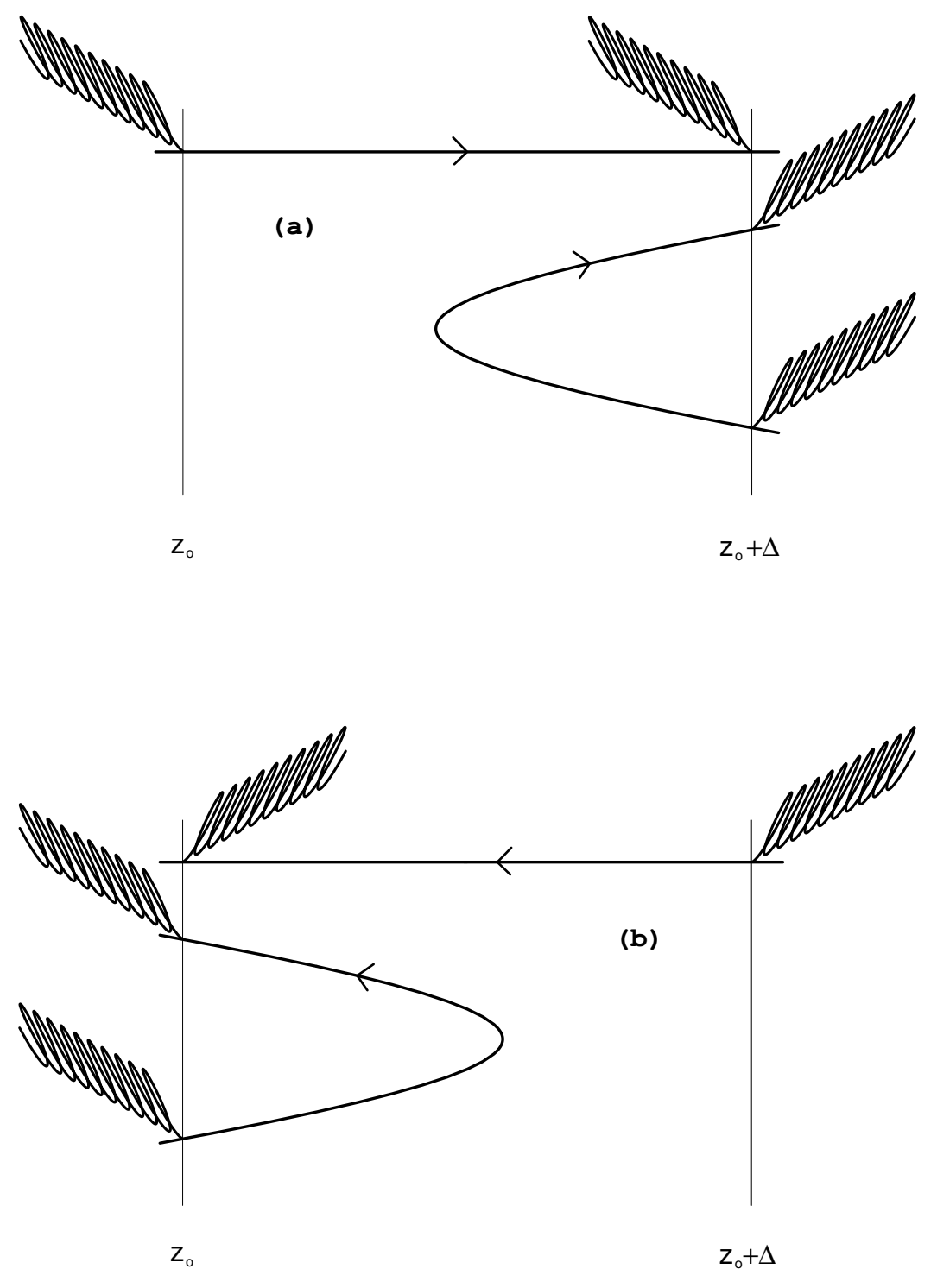

Fig. 1 

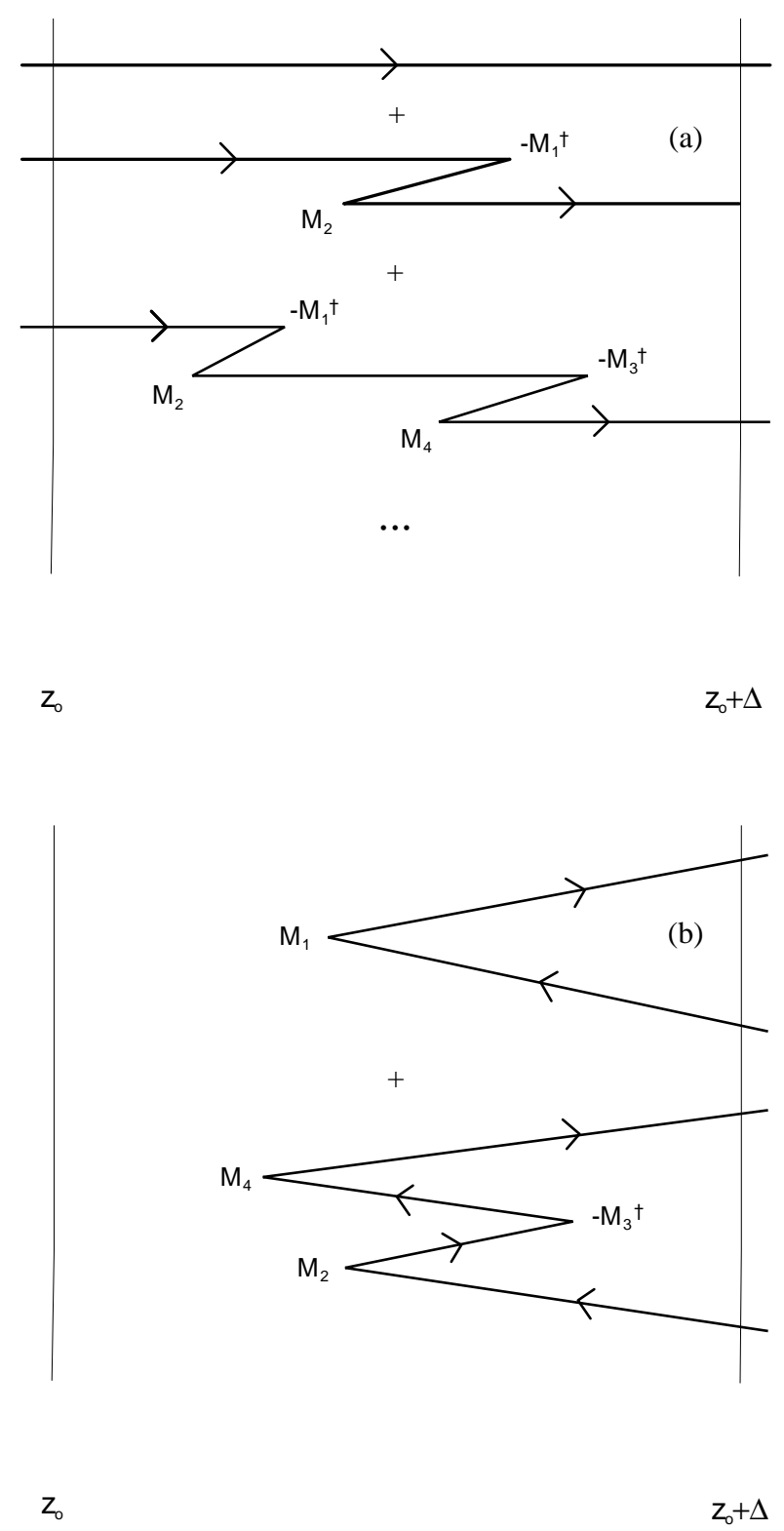

Fig. 2 

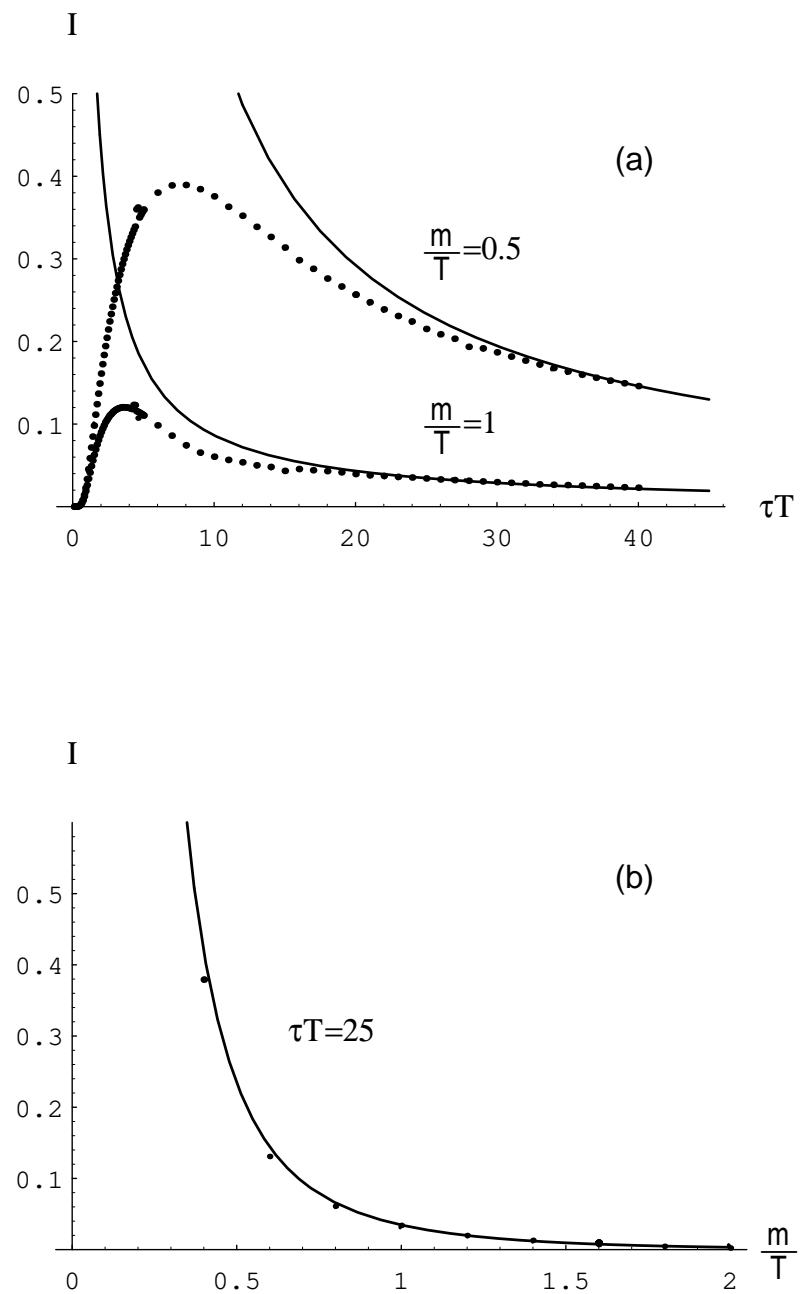

Fig. 3 


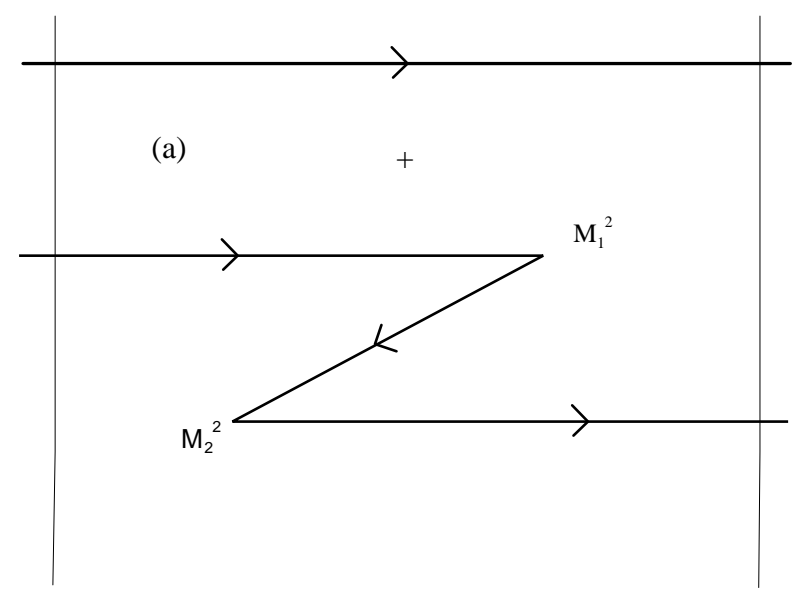

$\mathrm{z}_{\mathrm{o}}$

$\mathrm{z}_{0}+\Delta$

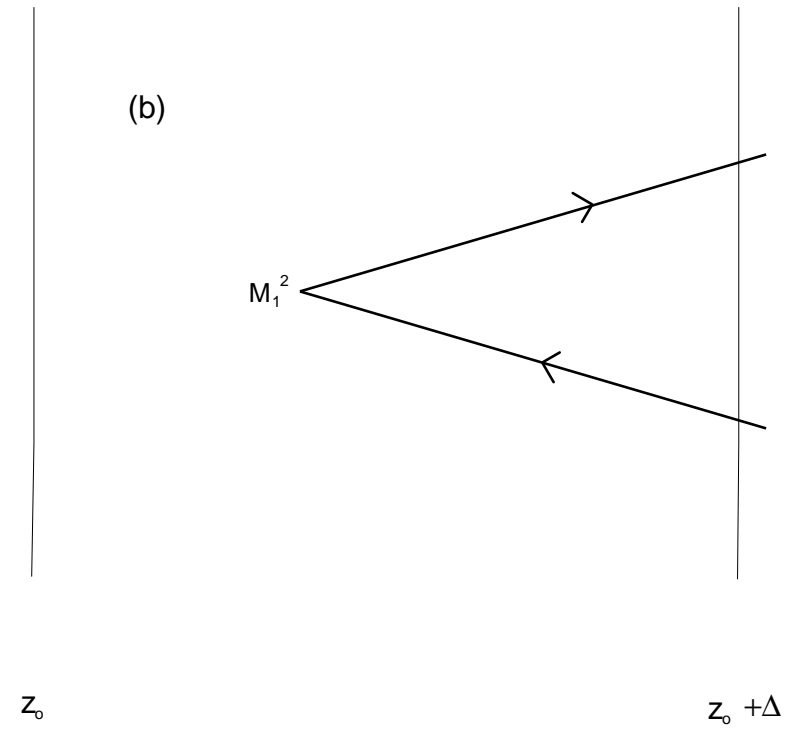

Fig. 4 

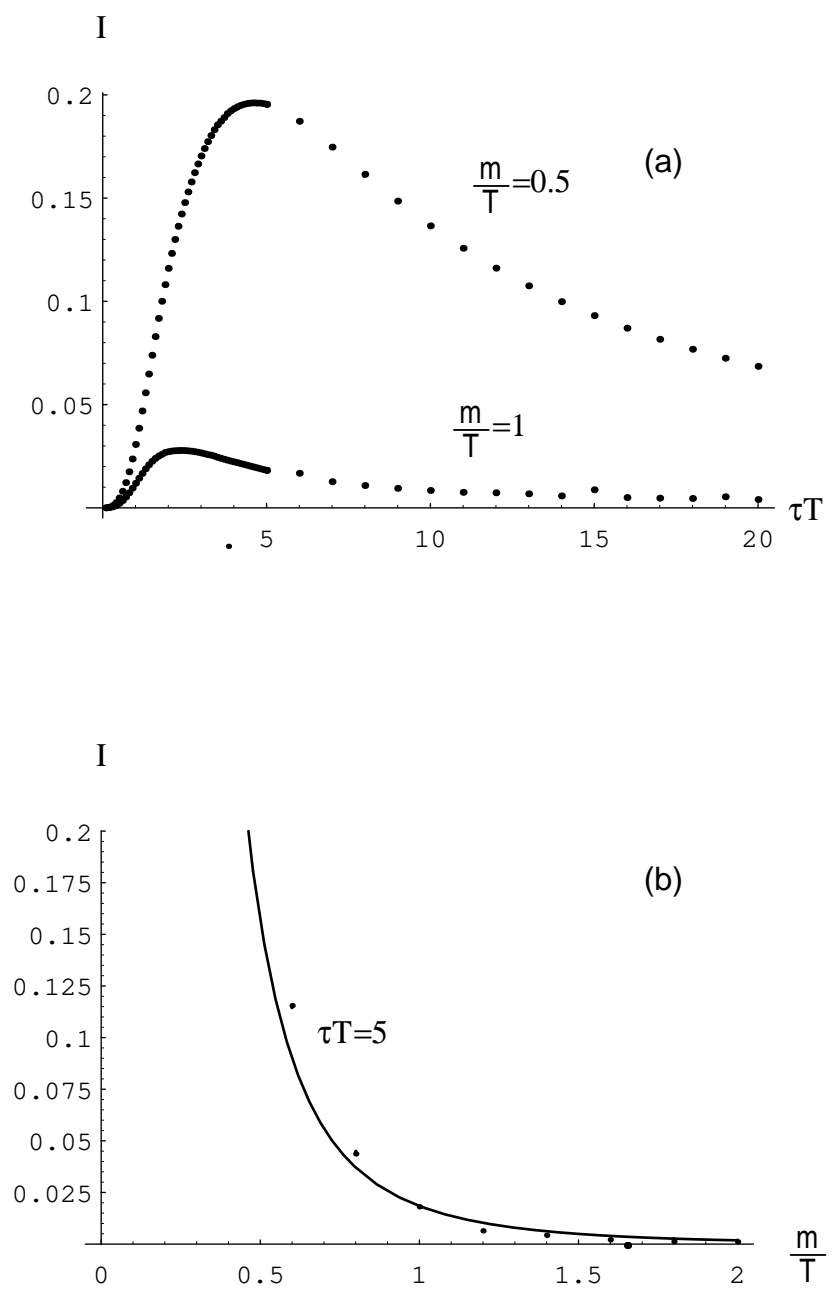

Fig. 5 